\title{
Synthesis of Analogue Structures of the para-Quinone Methide Moiety of Kendomycin
}

\section{Supporting Information}

\section{Martin P. Green, Stefan Pichlmair, Maria M. B. Marques, Harry J. Martin, Oliver Diwald, Thomas Berger and Johann Mulzer*}

\section{General Experimental}

All other solvents used HPLC grade. Column chromatography was performed with Merck silica gel (0.04-0.63 $\mu \mathrm{m}, 240-400$ mesh) under pressure. TLC was carried out with E. Merck silica gel 60-F254 plates. NMR spectra were recorded on either a Brucker Avance DPX $250 \mathrm{MHz}$ or $400 \mathrm{MHz}$. Unless otherwise stated, all NMR spectra were measured in $\mathrm{CDCl}_{3}$ solutions and referenced to the residual $\mathrm{CHCl}_{3}$ signal $\left({ }^{1} \mathrm{H}, \delta=7.27\right.$; ${ }^{13} \mathrm{C}, \delta=77.0$ ). All ${ }^{1} \mathrm{H}$ and ${ }^{1} \mathrm{C}$ shifts are given in ppm ( $\mathrm{s}=$ singlet; $\mathrm{d}=$ doublet; $\mathrm{t}=$ triplet; $\mathrm{q}$ $=$ quadruplet; $\mathrm{m}=$ multiplet; $\mathrm{br} \mathrm{s}=$ broad signal). Coupling constants $J$ are given in Hz. Mass spectra were measured on a MAT 900 (Finnegan-MAT, San Jose, CA). Highresolution mass spectra (HRMS) were taken with a Finnigan MAT 8230 with a resolution of 10,000 (Finnigan-MAT, San Jose, CA). The EPR experiment was performed at room temperature with a Bruker EMX 10/12 spectrometer operated in the X-band (9-10 GHz). In order to improve the signal-to-noise ratio 20 scans were accumulated in one spectrum.

1-(4-Methoxy-2,5-bis-methoxymethoxy-3-methyl-phenyl)-3-methyl-butan-2-ol (ㅁa)<smiles>COc1cc(Br)c(OC)c(OC)c1C</smiles>
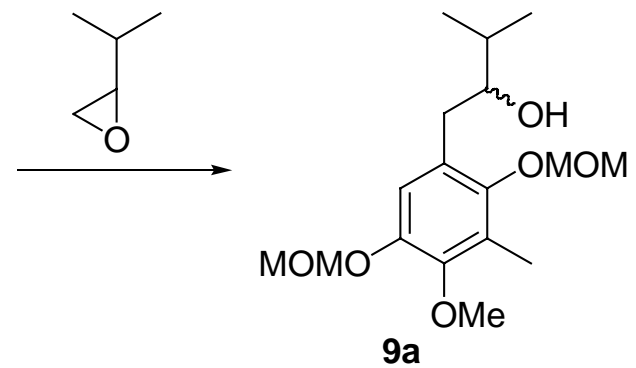

The aryl bromide $2 \mathrm{c}(2.00 \mathrm{~g}, 6.25 \mathrm{mmol})$ was dissolved in $10 \mathrm{~mL}$ of dry THF and $\mathrm{Mg}$ (163 $\mathrm{mg}, 6.25)$ was suspended in the reaction mixture. Iodine ( $2 \mathrm{mg}$ ) was added and the 
mixture was heated to reflux for $2 \mathrm{~h}$. The mixture was cooled to room temperature and transferred via cannula to a three necked flask containing $\mathrm{CuI}(20 \mathrm{mg}, 0.1 \mathrm{mmol})$ which was suspended in $2 \mathrm{~mL}$ of dry $\mathrm{THF}$ at $-50{ }^{\circ} \mathrm{C}$. The temperature was raised to $-30{ }^{\circ} \mathrm{C}$ and the mixture was stirred at this temperature for $30 \mathrm{~min}$. Afterwards, isobutene oxide (686 mg $8.0 \mathrm{mmol}$ ) was added at $-60{ }^{\circ} \mathrm{C}$ and the temperature was raised to $0{ }^{\circ} \mathrm{C}$ over $4 \mathrm{~h}$. The reaction mixture was quenched with $\mathrm{NH}_{4} \mathrm{Cl}(10 \mathrm{~mL})$ and extracted with diethyl $\mathrm{Et}_{2} \mathrm{O}(3 \mathrm{x}$ $10 \mathrm{~mL})$. The combined organic phases were washed with $\mathrm{H}_{2} \mathrm{O}(10 \mathrm{~mL})$, brine $(10 \mathrm{~mL})$ dried over $\mathrm{MgSO}_{4}$ and the solvent was removed by evaporation. The crude material was purified by $\mathrm{SiO}_{2}$ flash column chromatography using hexane and EtOAc (3:1) to afford 9a, as a colorless oil $(1.74 \mathrm{~g}, 85 \%) . \delta_{\mathrm{H}}\left(400 \mathrm{MHz} ; \mathrm{CDCl}_{3}\right) 6.85(1 \mathrm{H}, \mathrm{s}, \mathrm{CH}), 5.20(1 \mathrm{H}, \mathrm{d}$, $J=6 \mathrm{~Hz}, \mathrm{CHH}), 5.17(1 \mathrm{H}, \mathrm{d}, J=6 \mathrm{~Hz}, \mathrm{CHH}), 4.96(1 \mathrm{H}, \mathrm{d}, J=6 \mathrm{~Hz}, \mathrm{CHH}), 4.92(1 \mathrm{H}$, d, $J=6 \mathrm{~Hz}, \mathrm{CHH}), 3.81\left(3 \mathrm{H}, \mathrm{s}, \mathrm{CH}_{3}\right), 3.62\left(3 \mathrm{H}, \mathrm{s}, \mathrm{CH}_{3}\right), 3.62-3.56(1 \mathrm{H}, \mathrm{m}, \mathrm{CHOH})$, 3.52 (3 H, s, $\left.\mathrm{CH}_{3}\right), 2.75$ (1 H, d, J=3 Hz, CHCHAr), 2.74 (1 H, s, CHHAr), 2.36 (1 H, d, $J=5 \mathrm{~Hz}, \mathrm{OH}), 2.21\left(3 \mathrm{H}, \mathrm{s}, \mathrm{CH}_{3}\right), 1.80-1.72\left(1 \mathrm{H}, \mathrm{m}, \mathrm{CH}\left(\mathrm{CH}_{3}\right), 1.01(6 \mathrm{H}, \mathrm{d}, J=7 \mathrm{~Hz}\right.$, $\left.\mathrm{CH}_{3} \mathrm{CH}\right) ; \delta_{\mathrm{C}}\left(100 \mathrm{MHz} ; \mathrm{CDCl}_{3}\right) 150.5(\mathrm{C}), 147.8(\mathrm{C}), 147.4(\mathrm{C}), 128.5$ (C), 126.1(C), $116.2(\mathrm{CH}), 100.3\left(\mathrm{CH}_{2}\right), 96.0\left(\mathrm{CH}_{2}\right), 60.8\left(\mathrm{CH}_{3}\right), 57.9\left(\mathrm{CH}_{3}\right), 56.6\left(\mathrm{CH}_{3}\right), 35.6\left(\mathrm{CH}_{2}\right)$, $34.4(\mathrm{CH}), 19.2\left(2 \times \mathrm{CH}_{3}\right), 17.8(\mathrm{CH}), 10.8\left(\mathrm{CH}_{3}\right) ; \mathrm{m} / z(\mathrm{EI}) 328\left(\mathrm{M}^{+}, 26.3 \%\right), 296$ (36.5), 266 (29.8), 221 (100.0); $\left(\mathrm{C}_{17} \mathrm{H}_{28} \mathrm{O}_{6}\right.$ requires 328.1886. Found 328.1881).

1-(4-Methoxy-2,5-bis-methoxymethoxy-3-methyl-phenyl)-3-methyl-butan-2-one (10)<smiles>COc1cc(C[C@H](O)C(C)C)c(OC)c(C)c1OC</smiles>

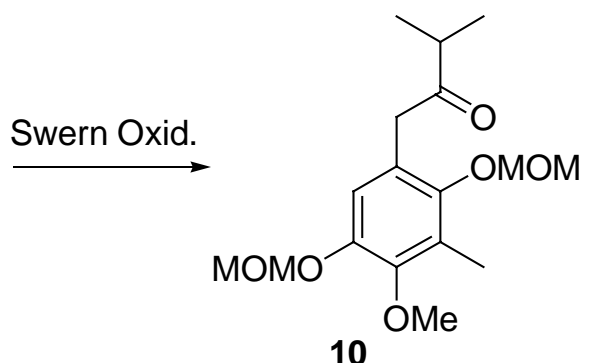

DMSO (1.3 mL, $18.3 \mathrm{mmol})$ was added dropwise to a solution of oxalyl chloride $(0.78$ $\mathrm{mL}, 9.1 \mathrm{mmol})$ in $\mathrm{CH}_{2} \mathrm{Cl}_{2}(10 \mathrm{~mL})$ at $-78{ }^{\circ} \mathrm{C}$. After the reaction mixture was stirred for one hour 9a (1.50 g, $4.57 \mathrm{mmol})$ was added slowly and stirring was continued for an additional $10 \mathrm{~min}$. After the dropwise addition of freshly distilled $\mathrm{NEt}_{3}(3.8 \mathrm{~mL}, 27.5$ mmol) a yellow slurry was formed and stirring was continued at $-78{ }^{\circ} \mathrm{C}$ for $30 \mathrm{~min}$. The reaction mixture was allowed to warm to room temperature $(45 \mathrm{~min})$, then hydrolyzed 
with $\mathrm{H}_{2} \mathrm{O}(10 \mathrm{~mL})$ and extracted with diethyl $\mathrm{Et}_{2} \mathrm{O}(3 \times 10 \mathrm{~mL})$. The combined organic layers were washed with saturated aqueous $\mathrm{NH}_{4} \mathrm{Cl}(5 \mathrm{~mL})$, saturated aqueous $\mathrm{NaHCO}_{3}(5$ $\mathrm{mL})$ and brine $(5 \mathrm{~mL})$, dried over $\mathrm{MgSO}_{4}$ and the solvent removed. The crude material was purified by $\mathrm{SiO}_{2}$ flash column chromatography using hexane and EtOAc (5:1) to afford 10, as a colorless oil $(1.33 \mathrm{~g}, 89 \%) . \delta_{\mathrm{H}}\left(400 \mathrm{MHz} ; \mathrm{CDCl}_{3}\right) 6.77(1 \mathrm{H}, \mathrm{s}, \mathrm{CH}), 5.17$ $\left(2 \mathrm{H}, \mathrm{s}, \mathrm{CH}_{2}\right), 4.86\left(2 \mathrm{H}, \mathrm{s}, \mathrm{CH}_{2}\right), 3.82\left(3 \mathrm{H}, \mathrm{s}, \mathrm{CH}_{3}\right), 3.77\left(2 \mathrm{H}, \mathrm{s}, \mathrm{CH}_{2}\right), 3.55(3 \mathrm{H}, \mathrm{s}$, $\left.\mathrm{CH}_{3}\right), 3.51\left(3 \mathrm{H}, \mathrm{s}, \mathrm{CH}_{3}\right), 2.73\left(1 \mathrm{H}, \mathrm{qq}, J=7 \mathrm{~Hz}\right.$, and $7 \mathrm{~Hz}, \mathrm{CH}\left(\mathrm{CH}_{3}\right), 2.22\left(3 \mathrm{H}, \mathrm{s}, \mathrm{CH}_{3}\right)$, $1.13\left(6 \mathrm{H}, \mathrm{d}, J=7 \mathrm{~Hz}, \mathrm{CH}_{3} \mathrm{CH}\right) ; \delta_{\mathrm{C}}\left(100 \mathrm{MHz} ; \mathrm{CDCl}_{3}\right) 212.6(\mathrm{C}), 150.3(\mathrm{C}), 147.3(\mathrm{C})$, $147.3(\mathrm{C}), 126.2(\mathrm{C}), 124.0(\mathrm{C}), 116.6(\mathrm{CH}), 100.1\left(\mathrm{CH}_{2}\right), 95.9\left(\mathrm{CH}_{2}\right), 60.8\left(\mathrm{CH}_{3}\right), 57.8$ $\left(\mathrm{CH}_{3}\right), 56.6\left(\mathrm{CH}_{3}\right), 43.1\left(\mathrm{CH}_{2}\right), 40.3(\mathrm{CH}), 18.8\left(2 \times \mathrm{CH}_{3}\right), 10.8\left(\mathrm{CH}_{3}\right) ; m / z(\mathrm{EI}) 326\left(\mathrm{M}^{+}\right.$, 96.0\%), 281 (36.5), 249 (62.9), 211 (41.6); $\left(\mathrm{C}_{17} \mathrm{H}_{26} \mathrm{O}_{6}\right.$ requires 326.1729. Found 326.1737).

\section{2-Isopropyl-6-methoxy-7-methyl-benzofuran-5-ol (미)}

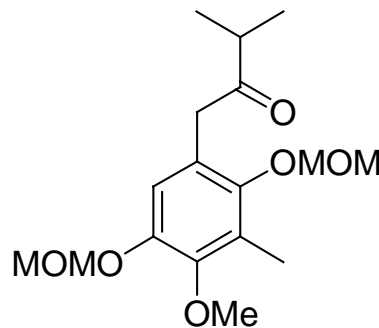

10

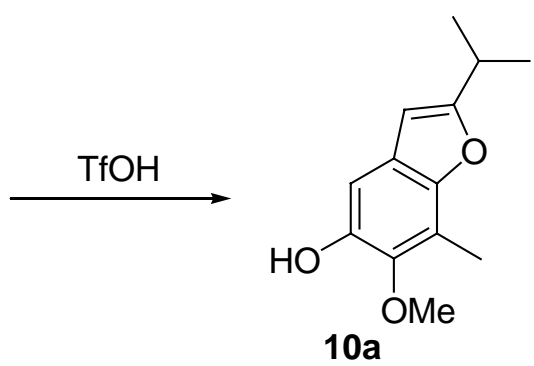

$10 \mathrm{a}$

Ketone 10 (1.00 g, $3.10 \mathrm{mmol})$ was dissolved in a solution of PhMe (200 mL) and EtOH (50 mL) and $3.0 \mathrm{~g}$ of $\mathrm{MS} 4 \AA$ were added. The suspension was heated to $80{ }^{\circ} \mathrm{C}$ and $\mathrm{CF}_{3} \mathrm{CO}_{3} \mathrm{H}(92 \mu \mathrm{L}, 1.24 \mathrm{mmol})$ was added. After $5 \mathrm{~min}$, the reaction flask was put into an ice bath to cool down to room temperature as quickly as possible. A saturated aq. solution of $\mathrm{NaHCO}_{3}(100 \mathrm{~mL})$ solution was added and the resulting mixture was filtered over a pad of Celite ${ }^{\circledR}$ to remove the molecular sieves. The layers were separated and the aq.layer was extracted with $\mathrm{Et}_{2} \mathrm{O}(3 \times 100 \mathrm{~mL})$, dried over $\mathrm{MgSO}_{4}$, and the solvent removed. The crude material was purified by $\mathrm{SiO}_{2}$ column chromatography using hexane and EtOAc (8:1) to afford 10a, as a brown oil (660 mg, $95 \%) \cdot v_{\max } / \mathrm{cm}^{-1}$ (film) 3750, 2966, 2934, $2873,1608,1463,1420,1352,1318,1265,1218,1189,1158,1131,1111,1070,991,942$ and $881 ; \delta_{\mathrm{H}}\left(400 \mathrm{MHz} ; \mathrm{CDCl}_{3}\right) ; 6.91(1 \mathrm{H}, \mathrm{s}, \mathrm{CH}), 6.23(1 \mathrm{H}, \mathrm{s}, \mathrm{CH}), 5.65(1 \mathrm{H}$, br s, $\mathrm{OH}), 3.85$ (3 H, s, OMe), $3.06\left(1 \mathrm{H}, \mathrm{qq}, J=7 \mathrm{~Hz}, \mathrm{CH}\left(\mathrm{CH}_{3}\right)_{2}\right), 2.48\left(3 \mathrm{H}, \mathrm{s}, \mathrm{CH}_{3}\right), 1.35$ (6 
$\left.\mathrm{H}, \mathrm{d}, J=7 \mathrm{~Hz}, \mathrm{CH}\left(\mathrm{CH}_{3}\right)_{3}\right) ; \delta_{\mathrm{C}}\left(100 \mathrm{MHz} ; \mathrm{CDCl}_{3}\right) 165.2(\mathrm{C}), 147.9(\mathrm{C}), 145.1(\mathrm{C}), 142.5$ (C), $124.1(\mathrm{C}), 113.8(\mathrm{C}), 102.1(\mathrm{CH}), 99.7(\mathrm{CH}), 61.3\left(\mathrm{CH}_{3}\right), 28.2(\mathrm{CH}), 20.4\left(2 \mathrm{x} \mathrm{CH}_{3}\right)$, $9.3\left(\mathrm{CH}_{3}\right) ; \mathrm{m} / z$ (EI) $220\left(\mathrm{M}^{+}, 64.9 \%\right), 205(100.0) ;\left(\mathrm{C}_{13} \mathrm{H}_{16} \mathrm{O}_{3}\right.$ requires 220.1099. Found 220.1096).

\section{2-Isopropyl-6-methoxy-5-methoxymethoxy-7-methyl-benzofuran (11)}
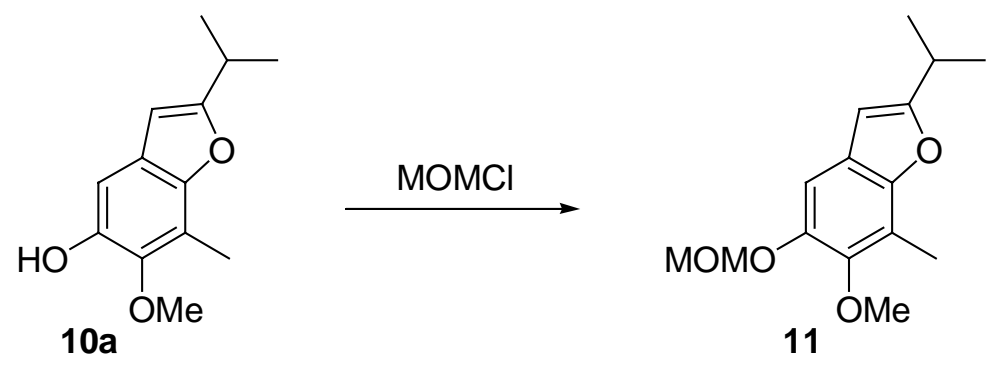

10a $(180 \mathrm{mg}, 0.82 \mathrm{mmol})$, was dissolved in dry DMF (2 mL) and $\mathrm{NaH}(60 \%$ in min. oil suspension, $66 \mathrm{mg}, 1.64 \mathrm{mmol}$ ) was added at $0{ }^{\circ} \mathrm{C}$ and the mixture stirred for $10 \mathrm{~min}$, forming a black suspension. Treatment with $\mathrm{MOMCl}(0.20 \mathrm{~mL}, 2.46 \mathrm{mmol})$ changed the color of the suspension to slightly brown. After 30 min a diethyl $\mathrm{Et}_{2} \mathrm{O}(3 \mathrm{~mL})$ and $\mathrm{H}_{2} \mathrm{O}(3$ $\mathrm{mL}$ ) were added. The layers were separated and the organic phase was extracted was diethyl $\mathrm{Et}_{2} \mathrm{O}(3 \times 3 \mathrm{~mL})$ and the combined organic layers were dried over $\mathrm{MgSO}_{4}$, filtered and distilled. The crude material was purified by flash column chromatography using hexane and EtOAc (5:1) to afford 11, as a colorless oil (180 mg, 83\%). $v_{\max } / \mathrm{cm}^{-1}$ (film) 2966, 2934, 2873, 1608, 1434, 1218, 1190, 1158, 1131, 1111, 1070, 991, 942, 881, 854, 793, 739 and 688; $\delta_{\mathrm{H}}\left(400 \mathrm{MHz} ; \mathrm{CDCl}_{3}\right) 7.09(1 \mathrm{H}, \mathrm{s}, \mathrm{CH}), 6.25(1 \mathrm{H}, \mathrm{d}, J=1 \mathrm{~Hz}, \mathrm{CH})$, $5.21\left(2 \mathrm{H}, \mathrm{s}, \mathrm{CH}_{2}\right), 3.84\left(3 \mathrm{H}, \mathrm{s}, \mathrm{CH}_{3}\right), 3.54\left(3 \mathrm{H}, \mathrm{s}, \mathrm{CH}_{3}\right), 3.05(1 \mathrm{H}, \mathrm{qqd}, J=7 \mathrm{~Hz}, 7 \mathrm{~Hz}$ and $1 \mathrm{~Hz}, \mathrm{CH}\left(\mathrm{CH}_{3}\right), 2.43\left(3 \mathrm{H}, \mathrm{s}, \mathrm{CH}_{3}\right), 1.33\left(6 \mathrm{H}, \mathrm{d}, J=7 \mathrm{~Hz},\left(\mathrm{CH}_{3}\right)_{2} \mathrm{CH}\right) ; \delta_{\mathrm{C}}(100 \mathrm{MHz}$; $\left.\mathrm{CDCl}_{3}\right)$ 165.6 (C), 149.7 (C), $147.3(\mathrm{C}), 146.0(\mathrm{C}), 123.9(\mathrm{C}), 115.6(\mathrm{C}), 105.5(\mathrm{CH})$, $100.2(\mathrm{CH}), 96.6\left(\mathrm{CH}_{2}\right), 61.4\left(\mathrm{CH}_{3}\right), 56.5\left(\mathrm{CH}_{3}\right), 28.7(\mathrm{CH}), 21.4\left(2 \times \mathrm{CH}_{3}\right), 9.5\left(\mathrm{CH}_{3}\right)$; $\mathrm{m} / \mathrm{z}$ (EI) $264\left(\mathrm{M}^{+}, 100 \%\right), 219(60.0) ;\left(\mathrm{C}_{15} \mathrm{H}_{20} \mathrm{O}_{4}\right.$ requires 264.1362. Found 264.1358).

\section{2-Isopropyl-6-methoxy-5-methoxymethoxy-4,7-dimethyl-benzofuran (11a)}



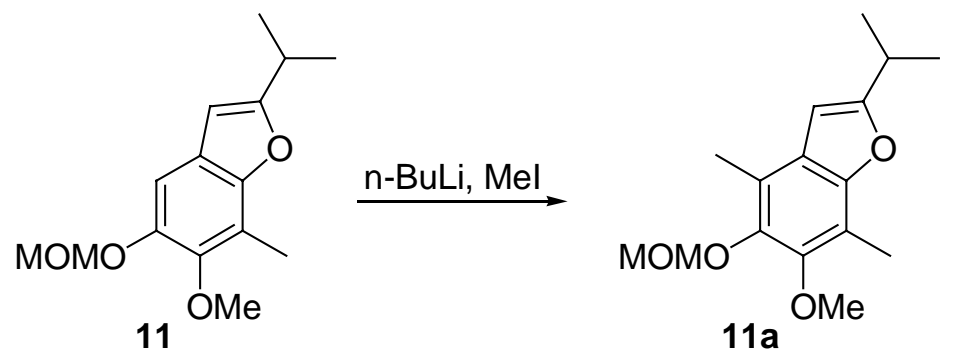

Benzofuran 11 (180 mg, $0.68 \mathrm{mmol})$ was dissolved in dry THF $(3 \mathrm{~mL})$ cooled to $-40{ }^{\circ} \mathrm{C}$ and $n$-Buli $(2.5 \mathrm{M}$ in hexanes, $0.80 \mathrm{~mL}, 2.0 \mathrm{mmol})$ was added. After one hour at $-30^{\circ}$ the solution was cooled to $-78{ }^{\circ} \mathrm{C}$ and methyl iodide $(0.43 \mathrm{~mL}, 68 \mathrm{mmol})$ was added. The reaction mixture was warmed to $-25^{\circ} \mathrm{C}$ over $2 \mathrm{~h}$ and finally quenched with saturated aqueous $\mathrm{NH}_{4} \mathrm{Cl}$ solution $(3 \mathrm{~mL})$. The reaction mixture was extracted with diethyl $\mathrm{Et}_{2} \mathrm{O}(3$ x $3 \mathrm{~mL}$ ), dried over $\mathrm{MgSO}_{4}$, filtered and the solvent was removed. The crude material was purified by flash column chromatography using hexane and EtOAc (7:1) to afford 11a, as a colorless oil (180 mg, 95\%). $v_{\max } / \mathrm{cm}^{-1}$ (film) 2964, 2926, 2361, 2344, 1654, 1560, 1458, 1389, 1338, 1160, 1118, 1093, 972, 1160, 1118, 1093, 972, 880, 738 and 610; $\delta_{\mathrm{H}}\left(400 \mathrm{MHz} ; \mathrm{CDCl}_{3}\right) 6.30(1 \mathrm{H}, \mathrm{d}, J=1 \mathrm{~Hz}, \mathrm{CH}), 5.09\left(2 \mathrm{H}, \mathrm{s}, \mathrm{CH}_{2}\right), 3.82(3 \mathrm{H}, \mathrm{s}$, $\left.\mathrm{CH}_{3}\right), 3.63\left(3 \mathrm{H}, \mathrm{s}, \mathrm{CH}_{3}\right), 3.06\left(1 \mathrm{H}, \mathrm{qqd}, J=7 \mathrm{~Hz}, 7 \mathrm{~Hz}\right.$ and $1 \mathrm{~Hz}, \mathrm{CH}\left(\mathrm{CH}_{3}\right), 2.41(3 \mathrm{H}$, s, $\left.\mathrm{CH}_{3}\right), 2.40\left(3 \mathrm{H}, \mathrm{s}, \mathrm{CH}_{3}\right), 1.34\left(6 \mathrm{H}, \mathrm{d}, J=7 \mathrm{~Hz}, \mathrm{CH}_{3} \mathrm{CH}\right) ; \delta_{\mathrm{C}}\left(100 \mathrm{MHz} ; \mathrm{CDCl}_{3}\right)$ 165.1 (C), 150.0 (C), 148.4 (C), 144.7 (C), 124.5(C), 120.2, (C), 112.9 (C), $100.0\left(\mathrm{CH}_{2}\right)$, 99.1 (CH), $61.2\left(\mathrm{CH}_{3}\right), 57.9\left(\mathrm{CH}_{3}\right), 28.7(\mathrm{CH}), 21.5\left(2 \times \mathrm{CH}_{3}\right), 13.2\left(\mathrm{CH}_{3}\right), 9.5\left(\mathrm{CH}_{3}\right) ; \mathrm{m} / z$ (EI) $278\left(\mathrm{M}^{+}, 91.7 \%\right), 233(100.0)$.

\section{2-Isopropyl-6-methoxy-4,7-dimethyl-benzofuran-5-ol (ㅁ)}

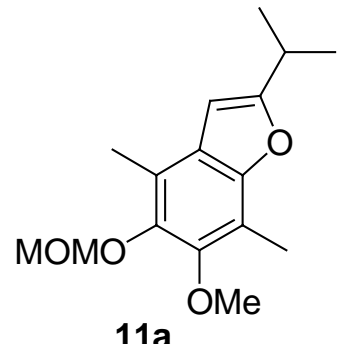

$11 \mathrm{a}$

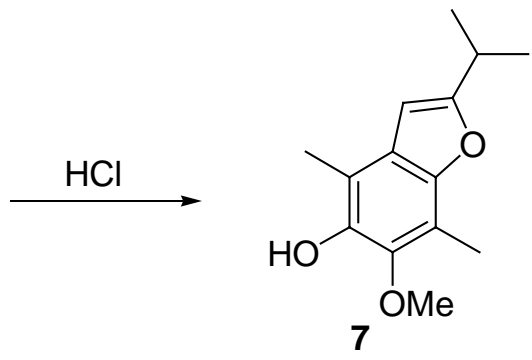

7

To a solution of 11a (150 mg, $0.54 \mathrm{mmol})$ in $\mathrm{MeOH}(3 \mathrm{~mL})$ was added aqueous $\mathrm{HCl}(1.0$ $\mathrm{M}, 0.3 \mathrm{~mL}, 0.3 \mathrm{mmol})$. The reaction was warmed to $50^{\circ} \mathrm{C}$. After $5 \mathrm{~h}, \mathrm{H}_{2} \mathrm{O}(3 \mathrm{~mL})$ was 
added and the product was extracted to $\mathrm{Et}_{2} \mathrm{O}(3 \times 5 \mathrm{~mL})$. The organic fractions were combined, washed with brine and dried over $\mathrm{MgSO}_{4}$. The solvent was removed and the crude material purified by flash column chromatography using hexane and EtOAc $(4: 1)$ to afford 7 as a light brown oil (123 mg, 96\%). $v_{\max } / \mathrm{cm}^{-1}$ (film) 3751, 2964, 2926, 1458, 1398, 1289, 1115, 1073, 880, 738 and 610; $\delta_{\mathrm{H}}\left(400 \mathrm{MHz} ; \mathrm{CDCl}_{3}\right) 6.29(1 \mathrm{H}, \mathrm{d}, J=1 \mathrm{~Hz}$, $\mathrm{CH}), 5.51(1 \mathrm{H}, \mathrm{s}, \mathrm{OH}), 3.83\left(3 \mathrm{H}, \mathrm{s}, \mathrm{CH}_{3}\right), 3.05(1 \mathrm{H}, \mathrm{qqd}, J=7 \mathrm{~Hz}, 7 \mathrm{~Hz}$ and $1 \mathrm{~Hz}$, $\mathrm{CH}\left(\mathrm{CH}_{3}\right), 2.43\left(3 \mathrm{H}, \mathrm{s}, \mathrm{CH}_{3}\right), 2.35\left(3 \mathrm{H}, \mathrm{s}, \mathrm{CH}_{3}\right), 1.34\left(6 \mathrm{H}, \mathrm{d}, J=7 \mathrm{~Hz}, \mathrm{CH}_{3} \mathrm{CH}\right) ; \delta_{\mathrm{C}}(100$ $\left.\mathrm{MHz} ; \mathrm{CDCl}_{3}\right)$ 165.0 (C), 147.4 (C), 143.0 (C), 142.8 (C), 124.8(C), 111.6, (C), 111.1 (C), $98.8(\mathrm{CH}), 61.8\left(\mathrm{CH}_{3}\right), 28.7(\mathrm{CH}), 21.4\left(2 \times \mathrm{CH}_{3}\right), 12.3\left(\mathrm{CH}_{3}\right), 9.6\left(\mathrm{CH}_{3}\right) ; \mathrm{m} / z(\mathrm{EI}) 234$ $\left(\mathrm{M}^{+}, 52.1 \%\right), 219(100.0)$.

\section{1-(2-Isopropyl-6-methoxy-5-methoxymethoxy-7-methyl-benzofuran-4-yl)-2-methyl-} propan-1-ol (15)

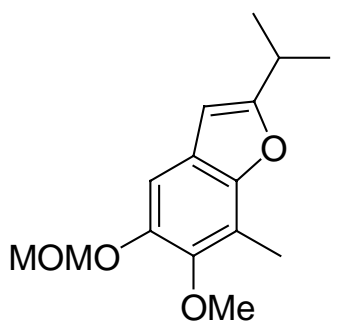

11

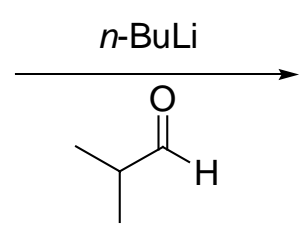

Benzofuran 11 (180 mg, $0.68 \mathrm{mmol})$ was dissolved in dry THF (3 mL) cooled to $-40{ }^{\circ} \mathrm{C}$ and $n$-Buli $(2.5 \mathrm{M}$ in hexanes, $0.80 \mathrm{~mL}, 2.0 \mathrm{mmol})$ was added. After one hour at $-30^{\circ}$ the solution was cooled to $-78{ }^{\circ} \mathrm{C}$ and isobutyraldehyde $(0.25 \mathrm{~mL}, 2.7 \mathrm{mmol})$ was added. The reaction mixture was warmed to $-25{ }^{\circ} \mathrm{C}$ over $2 \mathrm{~h}$ and finally quenched with saturated aqueous $\mathrm{NH}_{4} \mathrm{Cl}$ solution $(2 \mathrm{~mL})$. The reaction mixture was extracted with diethyl $\mathrm{Et}_{2} \mathrm{O}(3$ x $5 \mathrm{~mL}$ ), dried over $\mathrm{MgSO}_{4}$, filtered and the solvent was removed. The crude material was purified by $\mathrm{SiO}_{2}$ flash column chromatography using hexane and EtOAc (5:1) to afford 15, as a colorless oil $(205 \mathrm{mg}, 90 \%) . \delta_{\mathrm{H}}\left(400 \mathrm{MHz} ; \mathrm{CDCl}_{3}\right) 6.51(1 \mathrm{H}, \mathrm{d}, J=1 \mathrm{~Hz}$, $\mathrm{CH}), 5.12\left(2 \mathrm{H}, \mathrm{s}, \mathrm{CH}_{2}\right) 4.74(1 \mathrm{H}, \mathrm{dd}, J=9.5 \mathrm{~Hz}, 5.5 \mathrm{~Hz}, \mathrm{CHOH}), 3.80\left(3 \mathrm{H}, \mathrm{s}, \mathrm{CH}_{3}\right)$, $3.59\left(3 \mathrm{H}, \mathrm{s}, \mathrm{CH}_{3}\right), 3.05\left(1 \mathrm{H}, \mathrm{qq}, J=7 \mathrm{~Hz}, 7 \mathrm{~Hz}\right.$ and $1 \mathrm{~Hz}, \mathrm{CH}\left(\mathrm{CH}_{3}\right), 2.77(1 \mathrm{H}, \mathrm{d}, J=$ $5.5 \mathrm{~Hz}, \mathrm{OH}), 2.41\left(3 \mathrm{H}, \mathrm{s}, \mathrm{CH}_{3}\right), 2.35-2.25(1 \mathrm{H}, \mathrm{m}, \mathrm{CH}), 1.34(3 \mathrm{H}, \mathrm{d}, J=7 \mathrm{~Hz}$, $\left.\mathrm{CH}_{3} \mathrm{CH}\right), 1.33\left(3 \mathrm{H}, \mathrm{d}, J=7 \mathrm{~Hz}, \mathrm{CH}_{3} \mathrm{CH}\right), 1.18\left(3 \mathrm{H}, \mathrm{d}, J=7 \mathrm{~Hz}, \mathrm{CH}_{3} \mathrm{CH}\right), 0.74(3 \mathrm{H}, \mathrm{d}$, $\left.J=7 \mathrm{~Hz}, \mathrm{CH}_{3} \mathrm{CH}\right) ; \delta_{\mathrm{C}}\left(100 \mathrm{MHz} ; \mathrm{CDCl}_{3}\right) 165.1(\mathrm{C}), 150.6(\mathrm{C}), 147.9(\mathrm{C}), 144.6(\mathrm{C})$, 
$126.2(\mathrm{C}), 122.7(\mathrm{C}), 114.8(\mathrm{C}), 100.5\left(\mathrm{CH}_{2}\right), 100.2(\mathrm{CH}), 76.1(\mathrm{CH}), 61.1\left(\mathrm{CH}_{3}\right), 58.0$ $\left(\mathrm{CH}_{3}\right), 34.6(\mathrm{CH}), 28.6(\mathrm{CH}), 21.4\left(2 \times \mathrm{CH}_{3}\right), 20.1\left(\mathrm{CH}_{3}\right), 19.9\left(\mathrm{CH}_{3}\right) 9.5\left(\mathrm{CH}_{3}\right) ; \mathrm{m} / z(\mathrm{EI})$ $336\left(\mathrm{M}^{+}, 44.0 \%\right), 293$ (26.7), 274 (100), 233 (94.6); $\left(\mathrm{C}_{19} \mathrm{H}_{28} \mathrm{O}_{5}\right.$ requires 336.1937. Found $336.1942)$.

\section{2-Isopropyl-6-methoxy-4-(1-methoxy-2-methyl-propyl)-7-methyl-benzofuran-5-ol}

(8)
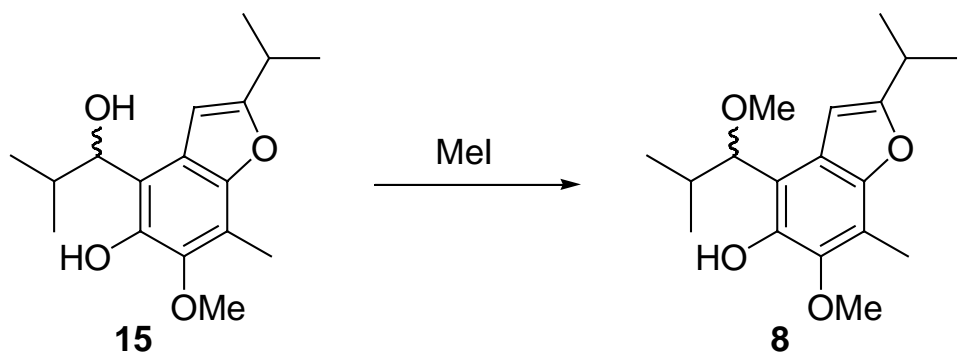

To a solution of $15(47 \mathrm{mg}, 0.14 \mathrm{mmol})$ in $\mathrm{MeOH}(3 \mathrm{~mL})$ was added aqueous $\mathrm{HCl}(1 \mathrm{~N})$ $(0.3 \mathrm{~mL}, 0.3 \mathrm{mmol})$. The reaction was warmed to $50^{\circ} \mathrm{C}$. After $5 \mathrm{~h}, \mathrm{H}_{2} \mathrm{O}(3 \mathrm{~mL})$ was added and the product was extracted to $\mathrm{Et}_{2} \mathrm{O}(3 \times 5 \mathrm{~mL})$. The organic fractions were combined, washed with brine and dried over $\mathrm{MgSO}_{4}$. The solvent was removed and the crude material purified by $\mathrm{SiO}_{2}$ flash column chromatography using hexane and ethyl acetate (4:1) to afford $\mathbf{8}$ as a colorless oil (33 mg, 77\%). $v_{\max } / \mathrm{cm}^{-1}$ (film) 2960, 2925, 1603, 1449, $1403,1356,1283,1104,944,894,816,738$ and $610 ; \delta_{\mathrm{H}}\left(400 \mathrm{MHz} ; \mathrm{CDCl}_{3}\right) 7.10(1 \mathrm{H}, \mathrm{br}$

s, OH), $6.28(1 \mathrm{H}, \mathrm{s}, \mathrm{CH}), 4.33(1 \mathrm{H}, \mathrm{d}, J=7.5 \mathrm{~Hz}, \mathrm{CHOMe}), 3.85\left(3 \mathrm{H}, \mathrm{s}, \mathrm{CH}_{3}\right), 3.34(3$ $\left.\mathrm{H}, \mathrm{s}, \mathrm{CH}_{3}\right), 3.04\left(1 \mathrm{H}, \mathrm{qq}, J=7 \mathrm{~Hz}\right.$ and $7 \mathrm{~Hz}, \mathrm{CH}\left(\mathrm{CH}_{3}\right), 2.43\left(3 \mathrm{H}, \mathrm{s}, \mathrm{CH}_{3}\right), 2.20-2.10(1$ $\mathrm{H}, \mathrm{m}, \mathrm{CH}), 1.34\left(6 \mathrm{H}, \mathrm{d}, J=7 \mathrm{~Hz}, \mathrm{CH}_{3} \mathrm{CH}\right), 1.07\left(3 \mathrm{H}, \mathrm{d}, J=7 \mathrm{~Hz}, \mathrm{CH}_{3} \mathrm{CH}\right), 0.85(3 \mathrm{H}$, d, $\left.J=7 \mathrm{~Hz}, \mathrm{CH}_{3} \mathrm{CH}\right) ; \delta_{\mathrm{C}}\left(100 \mathrm{MHz} ; \mathrm{CDCl}_{3}\right) 165.0(\mathrm{C}), 144.8(\mathrm{C}), 143.5(\mathrm{C}), 142.2(\mathrm{C})$, 123.2 (C), 114.0, (C), 99.1 (CH), $87.1(\mathrm{CH}), 61.3\left(\mathrm{CH}_{3}\right), 58.0\left(\mathrm{CH}_{3}\right), 34.3(\mathrm{CH}), 28.7$ $(\mathrm{CH}), 21.4\left(2 \times \mathrm{CH}_{3}\right), 19.7\left(\mathrm{CH}_{3}\right), 19.2\left(\mathrm{CH}_{3}\right) 9.5\left(\mathrm{CH}_{3}\right) ; \mathrm{m} / \mathrm{z}$ (EI) $306\left(\mathrm{M}^{+}, 19.3 \%\right), 278$, (21.8), 274 (100.0), 263 (63.2), 233 (67.6).

\section{2,5-Dihydroxy-2-isopropyl-4,7-dimethyl-2H-benzofuran-6-one (14)}




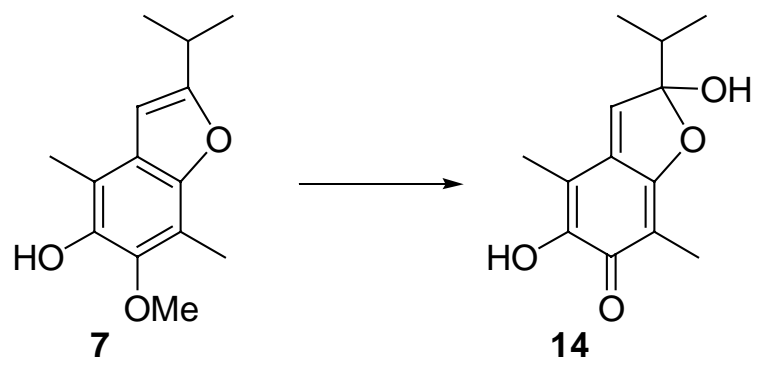

To a solution of benzofuran $7(14 \mathrm{mg}, 0.06 \mathrm{mmol})$ in DCM $(1 \mathrm{~mL})$ at $0^{\circ} \mathrm{C}$ was added a $0.2 \mathrm{M}$ solution of boron tribromide and lutidine (1:1) $(1.2 \mathrm{~mL}, 0.24 \mathrm{mmol})$ in DCM. After stirring for $10 \mathrm{~h}$ at $0-4^{\circ} \mathrm{C}$, methanol was added and the reaction stirred at room temperature. After $5 \mathrm{~h}, \mathrm{H}_{2} \mathrm{O}(3 \mathrm{~mL})$ and $\mathrm{Et}_{2} \mathrm{O}(5 \mathrm{~mL})$ were added and the layers separated. The aqueous phase was extracted with $\mathrm{Et}_{2} \mathrm{O}(3 \times 5 \mathrm{~mL})$. The organic fractions were combined, washed with brine and dried over $\mathrm{MgSO}_{4}$. The solvent was removed to give a crude sample of catechol 18 which was used directly in the next reaction.

To a solution of crude catechol $\mathbf{1 8}$ in DCM $(2 \mathrm{~mL})$ at $-78^{\circ} \mathrm{C}$ was added a solution of dimethyldioxirane $(0.09 \mathrm{M}$ in acetone, $0.73 \mathrm{~mL}, 0.07 \mathrm{mmol})$. After stirring for $20 \mathrm{~min}$ at this temperature, the reagent and solvent were removed by evaporation. The crude product was purified by $\mathrm{SiO}_{2}$ flash column chromatography using hexane and $\mathrm{Et}_{2} \mathrm{O}$ (4:1) to afford para-quinone methide $\mathbf{1 4}$ as a yellow oil (10 mg, 73\%). $v_{\max } / \mathrm{cm}^{-1}$ (film) 3356 , 2969, 2360, 2343, 1671, 1613, 1584, 1568, 1381, 1329, 1263, 1116, 1091, 926, 891, 788, 737 and 610; $\delta_{\mathrm{H}}\left(400 \mathrm{MHz} ; \mathrm{CDCl}_{3}\right) 7.18(1 \mathrm{H}, \mathrm{br} \mathrm{s}, \mathrm{OH}), 6.63(1 \mathrm{H}, \mathrm{s}, \mathrm{CH}), 4.09(1 \mathrm{H}, \mathrm{br}$ s, $\mathrm{OH}), 2.23\left(1 \mathrm{H}, \mathrm{qq}, J=7 \mathrm{~Hz}\right.$ and $7 \mathrm{~Hz}, \mathrm{CH}\left(\mathrm{CH}_{3}\right), 2.03\left(3 \mathrm{H}, \mathrm{s}, \mathrm{CH}_{3}\right), 1.90(3 \mathrm{H}, \mathrm{s}$, $\left.\mathrm{CH}_{3}\right), 1.07\left(3 \mathrm{H}, \mathrm{d}, J=7 \mathrm{~Hz}, \mathrm{CH}_{3} \mathrm{CH}\right), 0.99\left(3 \mathrm{H}, \mathrm{d}, J=7 \mathrm{~Hz}, \mathrm{CH}_{3} \mathrm{CH}\right) ; \delta_{\mathrm{C}}(100 \mathrm{MHz}$; $\left.\mathrm{CDCl}_{3}\right) 182.2(\mathrm{C}), 166.9(\mathrm{C}), 145.9(\mathrm{C}), 135.8(\mathrm{CH}), 133.9(\mathrm{C}), 116.8(\mathrm{C}), 106.3(\mathrm{C})$, 104.6 (CH), $35.3\left(\mathrm{CH}_{3}\right), 17.0\left(\mathrm{CH}_{3}\right), 16.4\left(\mathrm{CH}_{3}\right), 10.0\left(\mathrm{CH}_{3}\right), 7.0\left(\mathrm{CH}_{3}\right) ; \mathrm{m} / z(\mathrm{EI}) 236$ $\left(\mathrm{M}^{+}, 32.8 \%\right), 193$ (100.0); $\left(\mathrm{C}_{13} \mathrm{H}_{16} \mathrm{O}_{4}\right.$ requires 236.1049. Found 236.1044).

\section{2,5-Dihydroxy-2-isopropyl-4-(1-methoxy-2-methyl-propyl)-7-methyl-2H- benzofuran-6-one (17)}




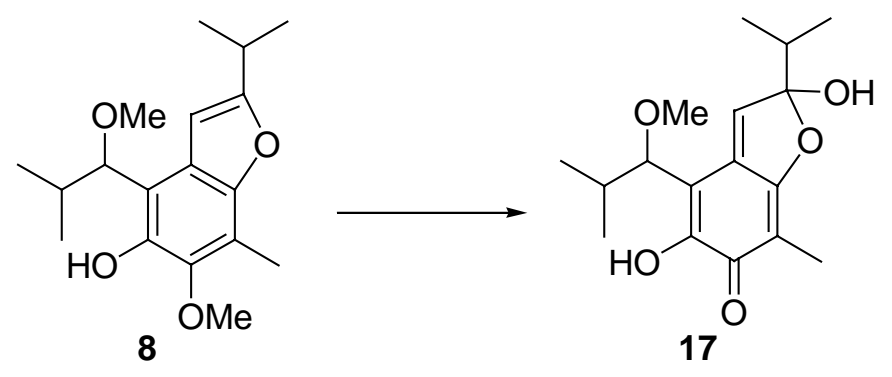

To a solution of benzofuran $8(9 \mathrm{mg}, 0.03 \mathrm{mmol})$ in DCM $(1 \mathrm{~mL})$ at $0^{\circ} \mathrm{C}$ was added a 0.2 M solution of boron tribromide and lutidine (1:1) $(0.60 \mathrm{~mL}, 0.12 \mathrm{mmol})$ in DCM. After stirring for $10 \mathrm{~h}$ at $0-4^{\circ} \mathrm{C}$, methanol was added and the reaction stirred at room temperature. After $5 \mathrm{~h}, \mathrm{H}_{2} \mathrm{O}(3 \mathrm{~mL})$ and $\mathrm{Et}_{2} \mathrm{O}(5 \mathrm{~mL})$ were added and the layers separated. The aqueous phase was extracted with $\mathrm{Et}_{2} \mathrm{O}(3 \times 5 \mathrm{~mL})$. The organic fractions were combined, washed with brine and dried over $\mathrm{MgSO}_{4}$. The solvent was removed to give a crude sample of catechol 19 which was used directly in the next reaction.

To a solution of crude catechol 19 in DCM $(2 \mathrm{~mL})$ at $-78^{\circ} \mathrm{C}$ was added a solution of dimethyldioxirane $(0.9 \mathrm{M}$ in acetone, $0.39 \mathrm{~mL}, 0.035 \mathrm{mmol})$. After stirring for $20 \mathrm{~min}$ at this temperature, the reagent and solvent were removed by evaporation. The crude product was purified by $\mathrm{SiO}_{2}$ flash column chromatography using hexane and ethyl acetate (6:1) to afford both diasteroisomers of para-quinone methide $\mathbf{1 7}$ as a yellow oil (6 $\mathrm{mg}, 65 \%$ ). $v_{\max } / \mathrm{cm}^{-1}$ (film) 3331, 2966, 2926, 1611, 1378, 1332, 1092, 920, 738 and 610; $\delta_{\mathrm{H}}\left(400 \mathrm{MHz} ; \mathrm{CDCl}_{3}\right) 7.46(1 \mathrm{H}, \mathrm{s}, \mathrm{OH}), 7.06$ and $7.05(1 \mathrm{H}, \mathrm{s}, \mathrm{CH}), 4.20$ and $4.18(1 \mathrm{H}$, d, $J=9 \mathrm{~Hz}, \mathrm{CHOMe}$ ), 3.26 and $3.22(3 \mathrm{H}, \mathrm{s}, \mathrm{OMe}), 3.00$ and $3.97(1 \mathrm{H}, \mathrm{s}, \mathrm{OH}), 2.26(1$ $\mathrm{H}, \mathrm{qq}, J=7 \mathrm{~Hz}$ and $\left.7 \mathrm{~Hz}, \mathrm{CHCH}_{3}\right), 2.2 .02-2.19\left(1 \mathrm{H}, \mathrm{m}, \mathrm{CHCH}_{3}\right), 1.94\left(3 \mathrm{H}, \mathrm{s}, \mathrm{CH}_{3}\right)$, 1.02 and $1.00\left(3 \mathrm{H}, \mathrm{d}, J=7 \mathrm{~Hz}, \mathrm{CH}_{3} \mathrm{CH}\right), 0.99\left(3 \mathrm{H}, \mathrm{d}, J=7 \mathrm{~Hz}, \mathrm{CH}_{3} \mathrm{CH}\right), 0.93$ and 0.92 $\left(3 \mathrm{H}, \mathrm{d}, J=7 \mathrm{~Hz}, \mathrm{CH}_{3} \mathrm{CH}\right), 0.76$ and $0.75\left(3 \mathrm{H}, \mathrm{d}, J=7 \mathrm{~Hz}, \mathrm{CH}_{3} \mathrm{CH}\right) ;{ }^{13} \mathrm{C}-\mathrm{NMR}\left(\mathrm{CDCl}_{3}\right.$, $100 \mathrm{MHz}$, quaternary carbons missing after 20000 scans): $\delta_{\mathrm{C}}\left(100 \mathrm{MHz} ; \mathrm{CDCl}_{3}\right) 138.9$ and $138.8(\mathrm{CH}), 100.2(\mathrm{C}), 83.2$ and $83.0(\mathrm{CH}), 57.7$ and $57.3\left(\mathrm{CH}_{3}\right), 36.3(\mathrm{CH}), 33.7$ and 33.6 $(\mathrm{CH}), 20.0$ and $19.9\left(\mathrm{CH}_{3}\right), 19.2$ and $19.1\left(\mathrm{CH}_{3}\right), 18.1$ and $18.0\left(\mathrm{CH}_{3}\right), 17.3$ and 17.2 $\left(\mathrm{CH}_{3}\right), 7.99\left(\mathrm{CH}_{3}\right) ; \mathrm{m} / \mathrm{z}$ (EI) $308\left(\mathrm{M}^{+}, 10.3 \%\right), 265$ (100.0), 205 (55.0); $\left(\mathrm{C}_{17} \mathrm{H}_{24} \mathrm{O}_{5}\right.$ requires 308.1624. Found 308.1617). 


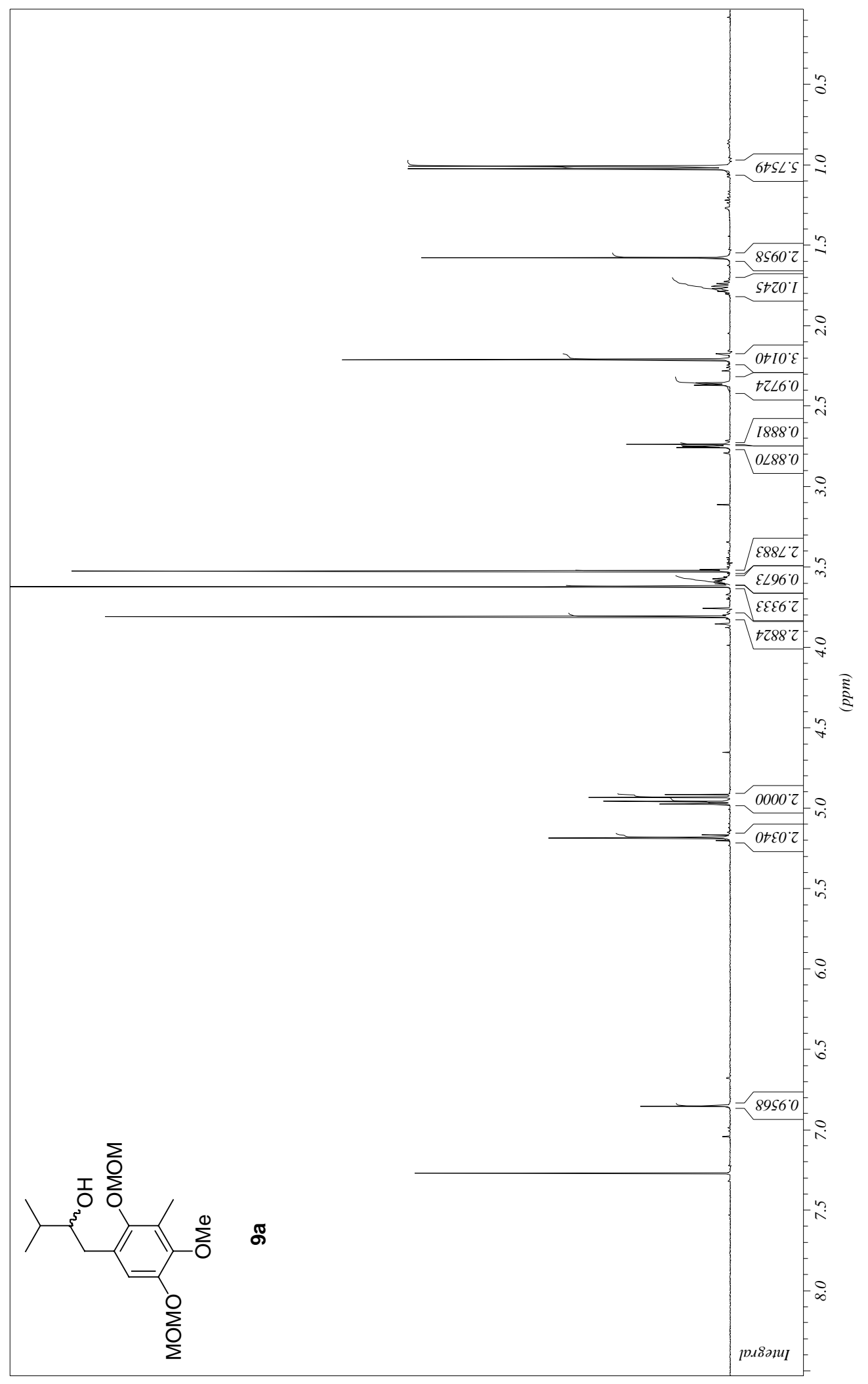

$\frac{0}{n}$ 


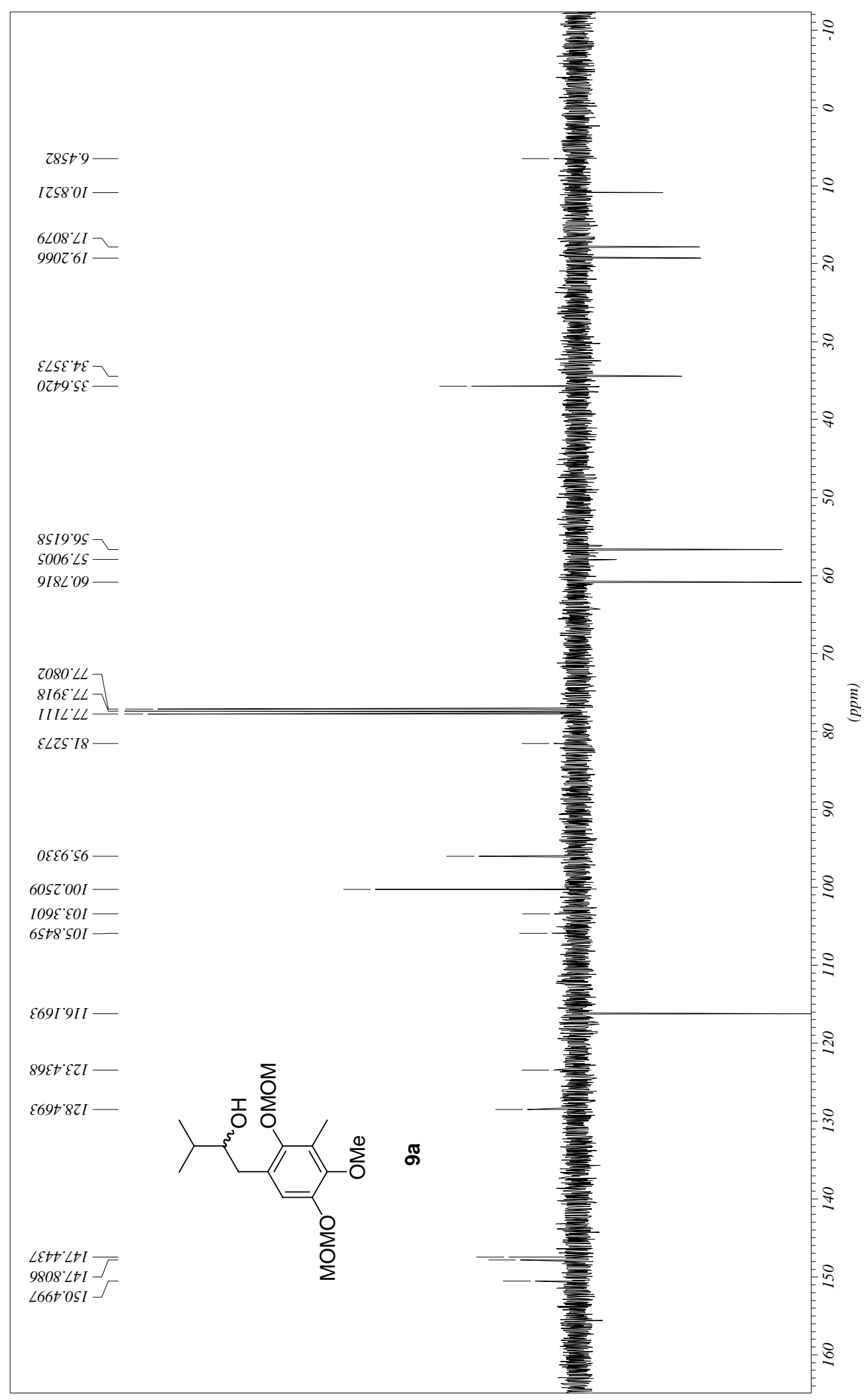




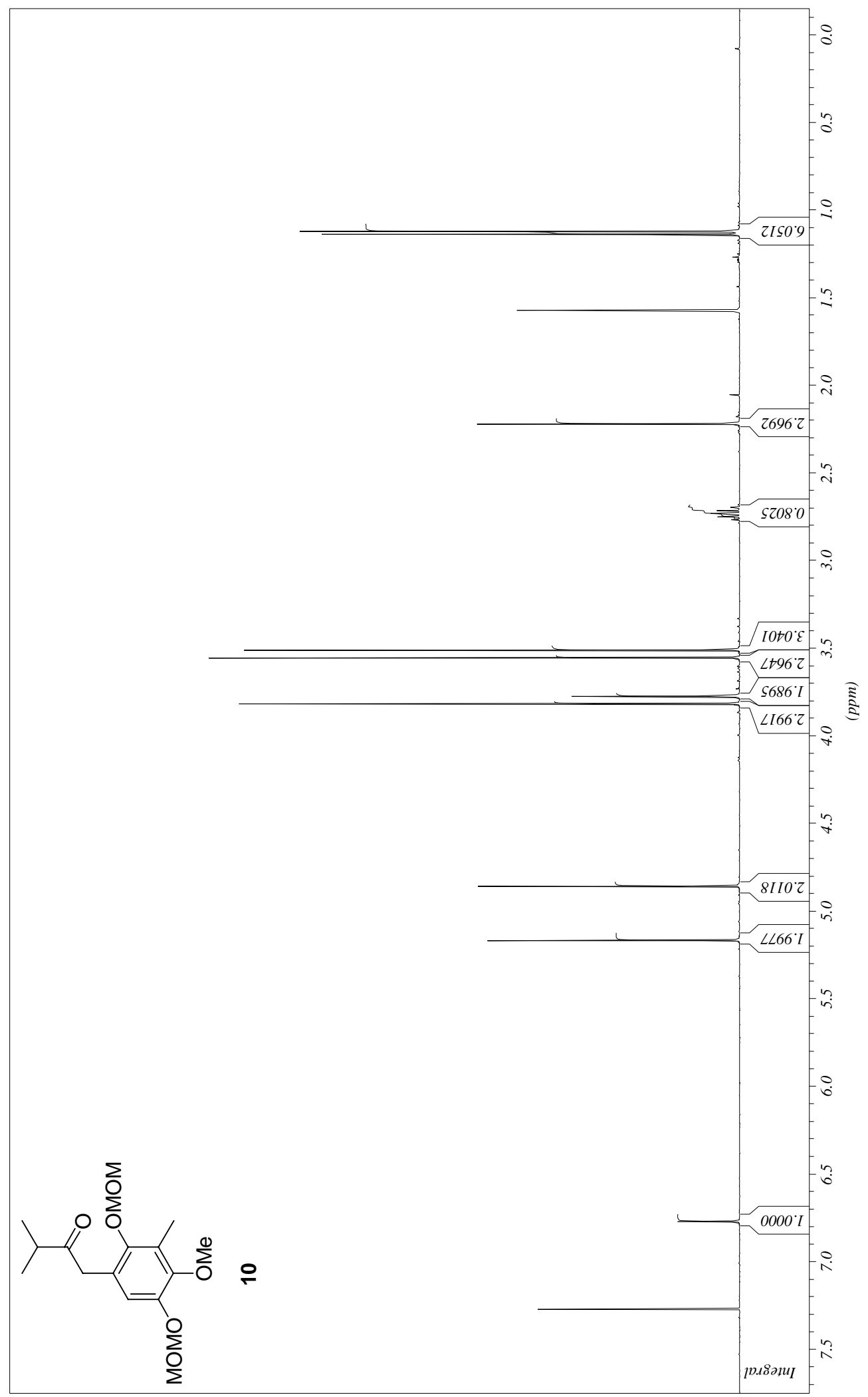




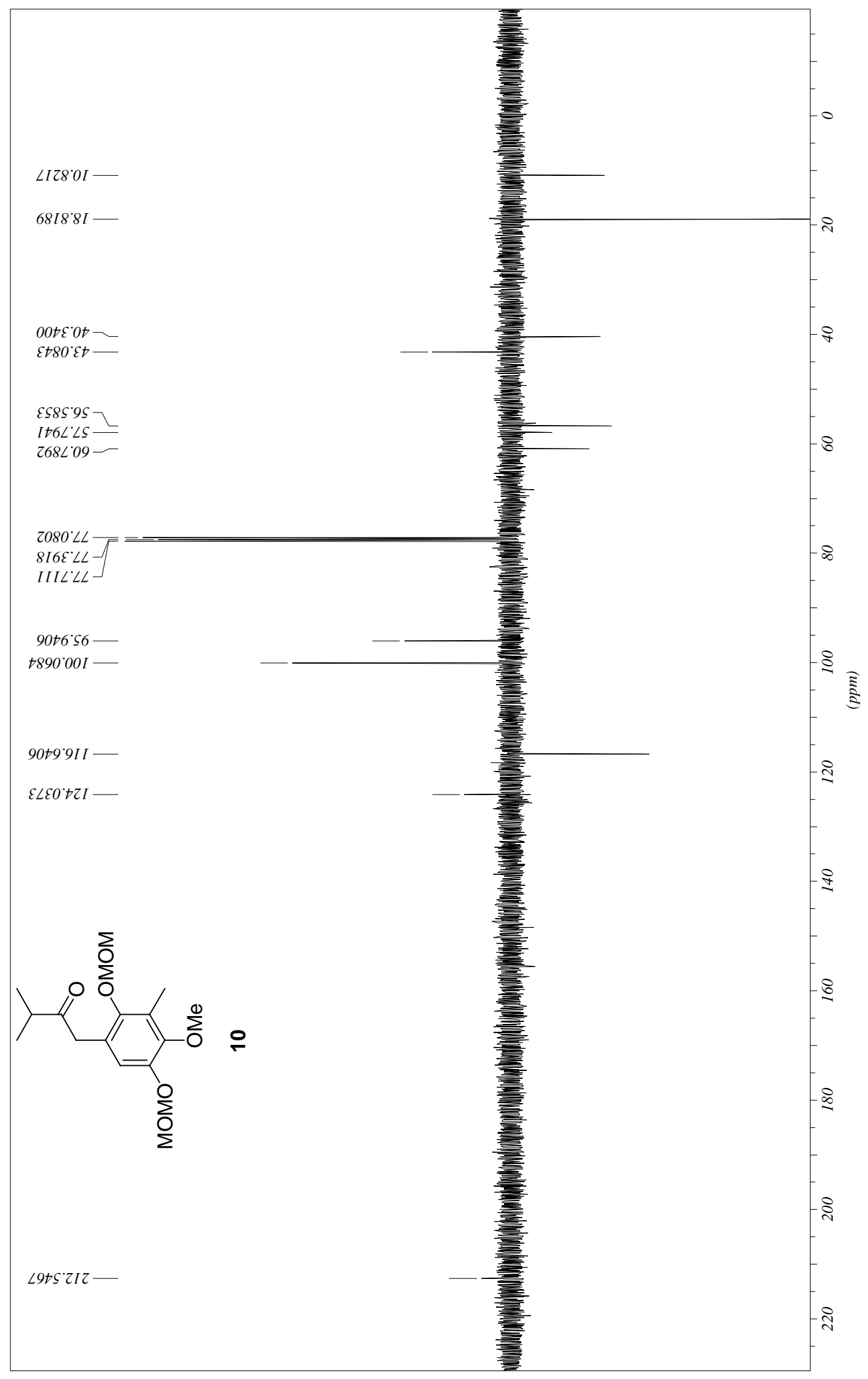




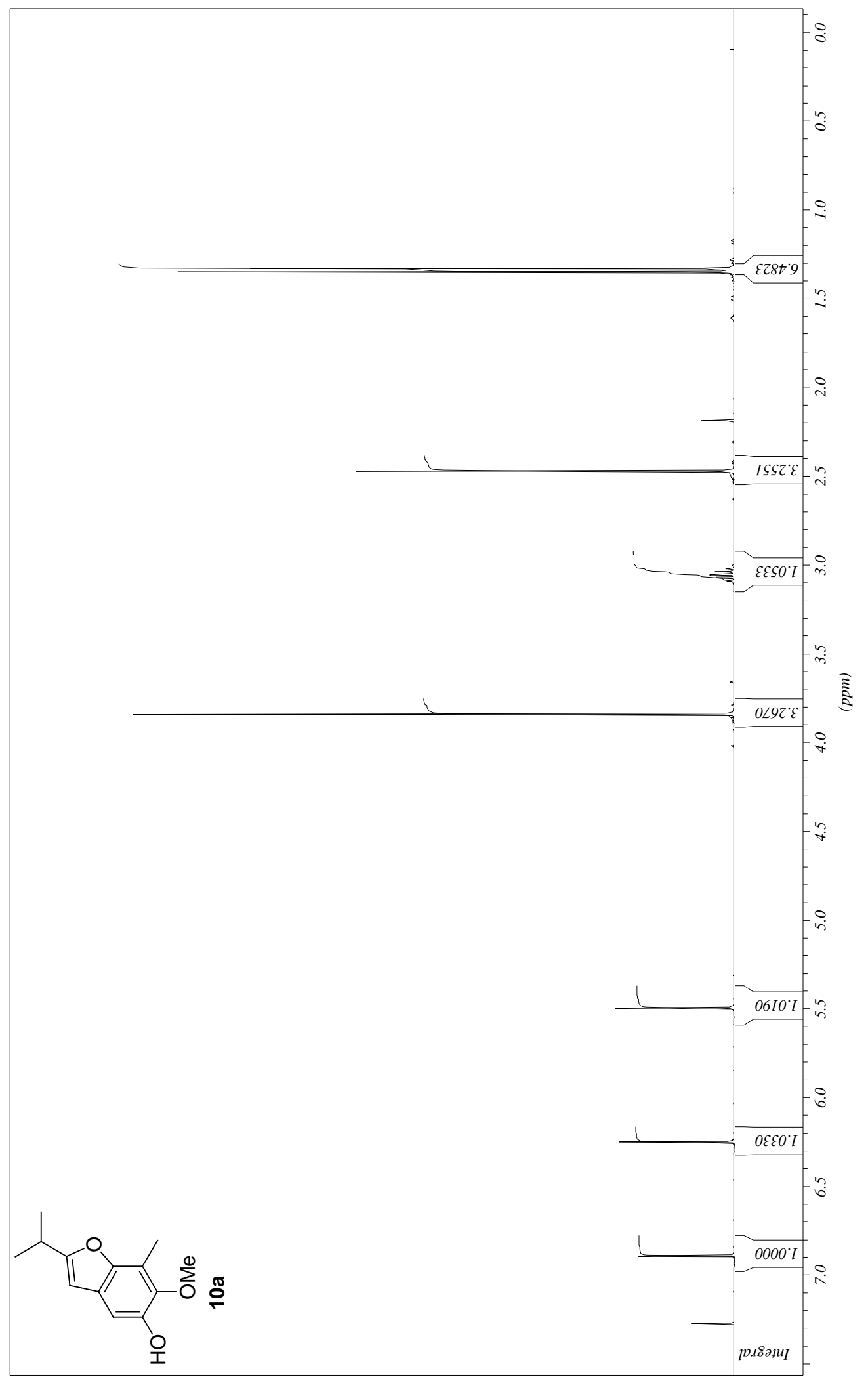




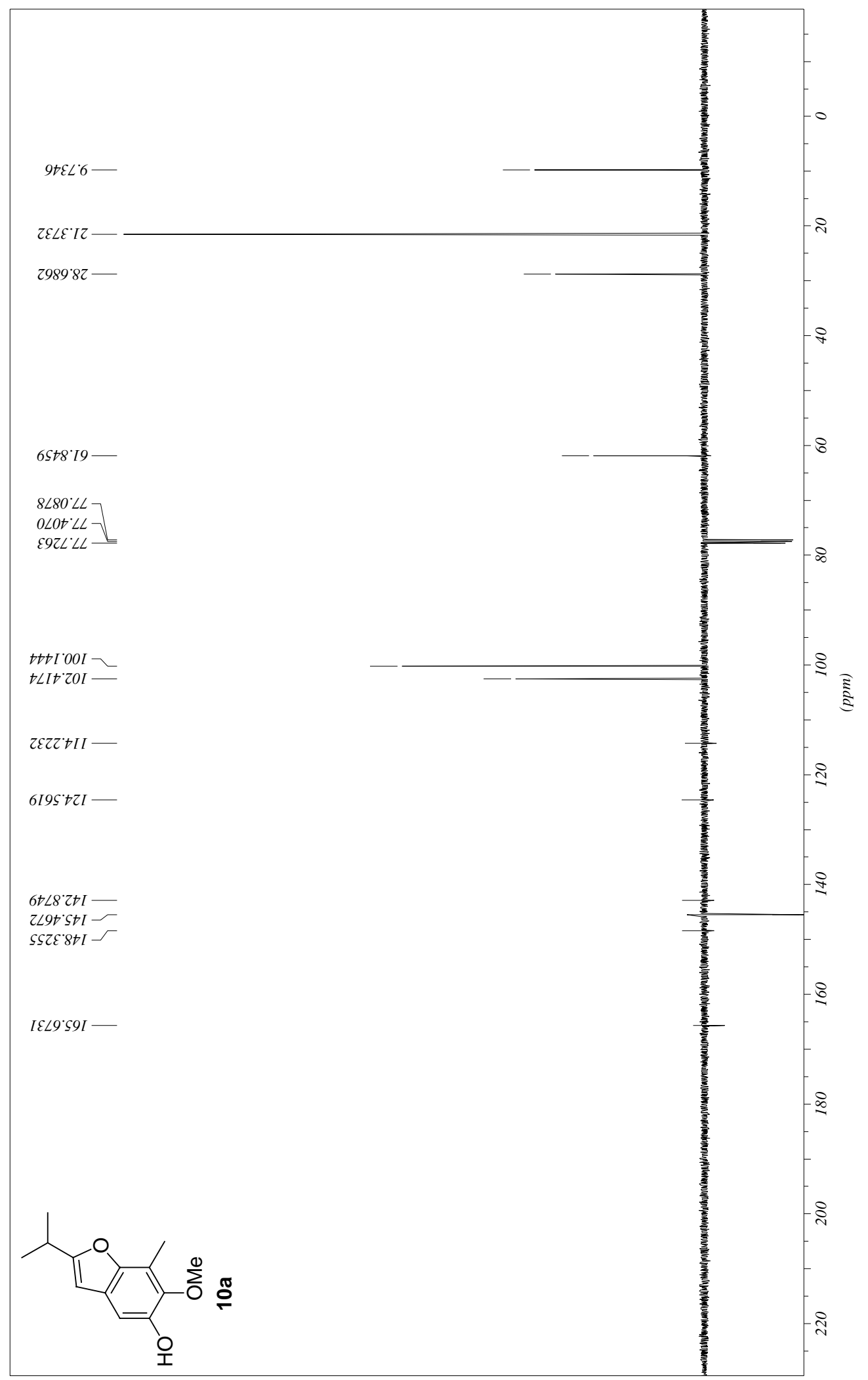




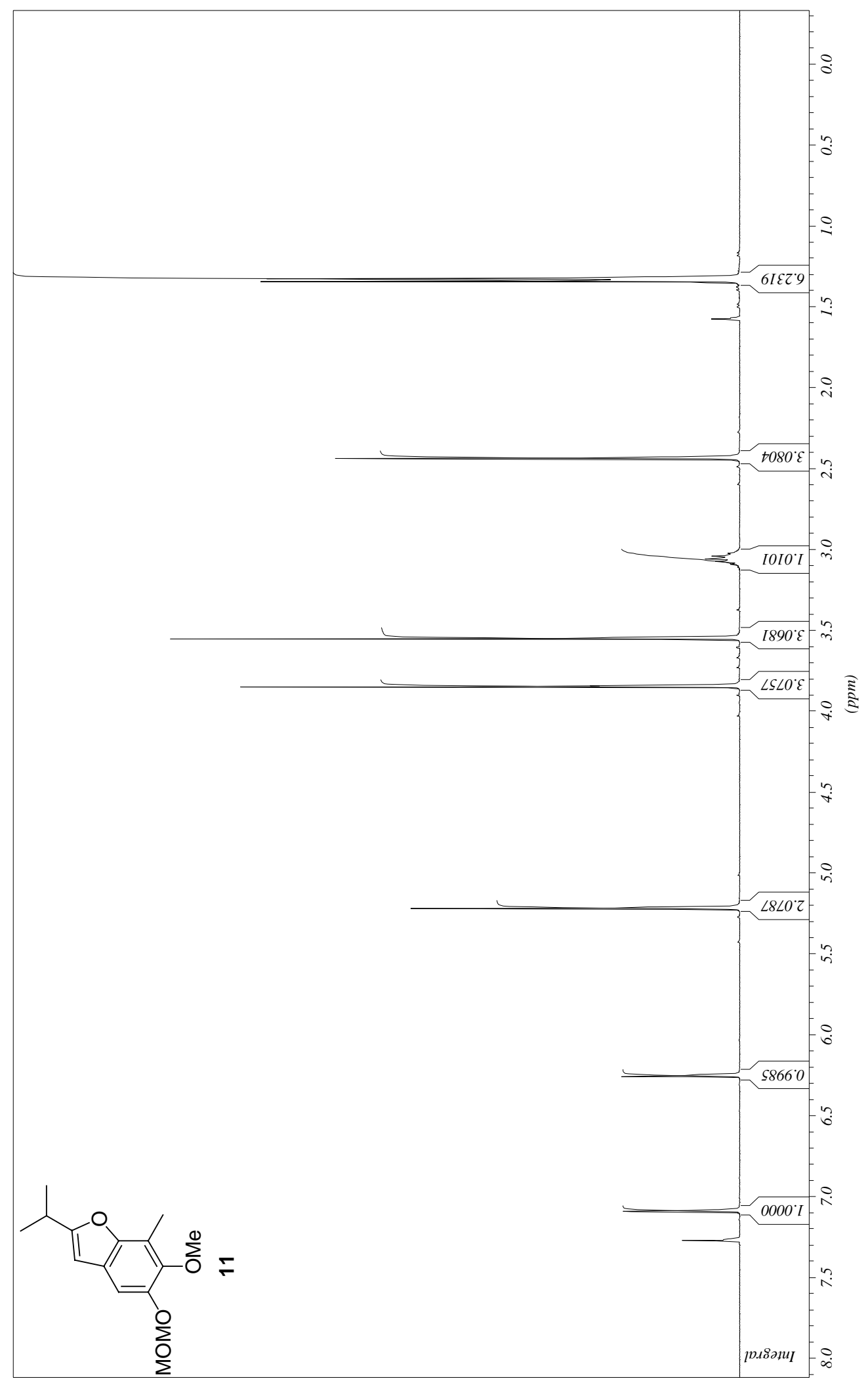

$\frac{6}{n}$ 


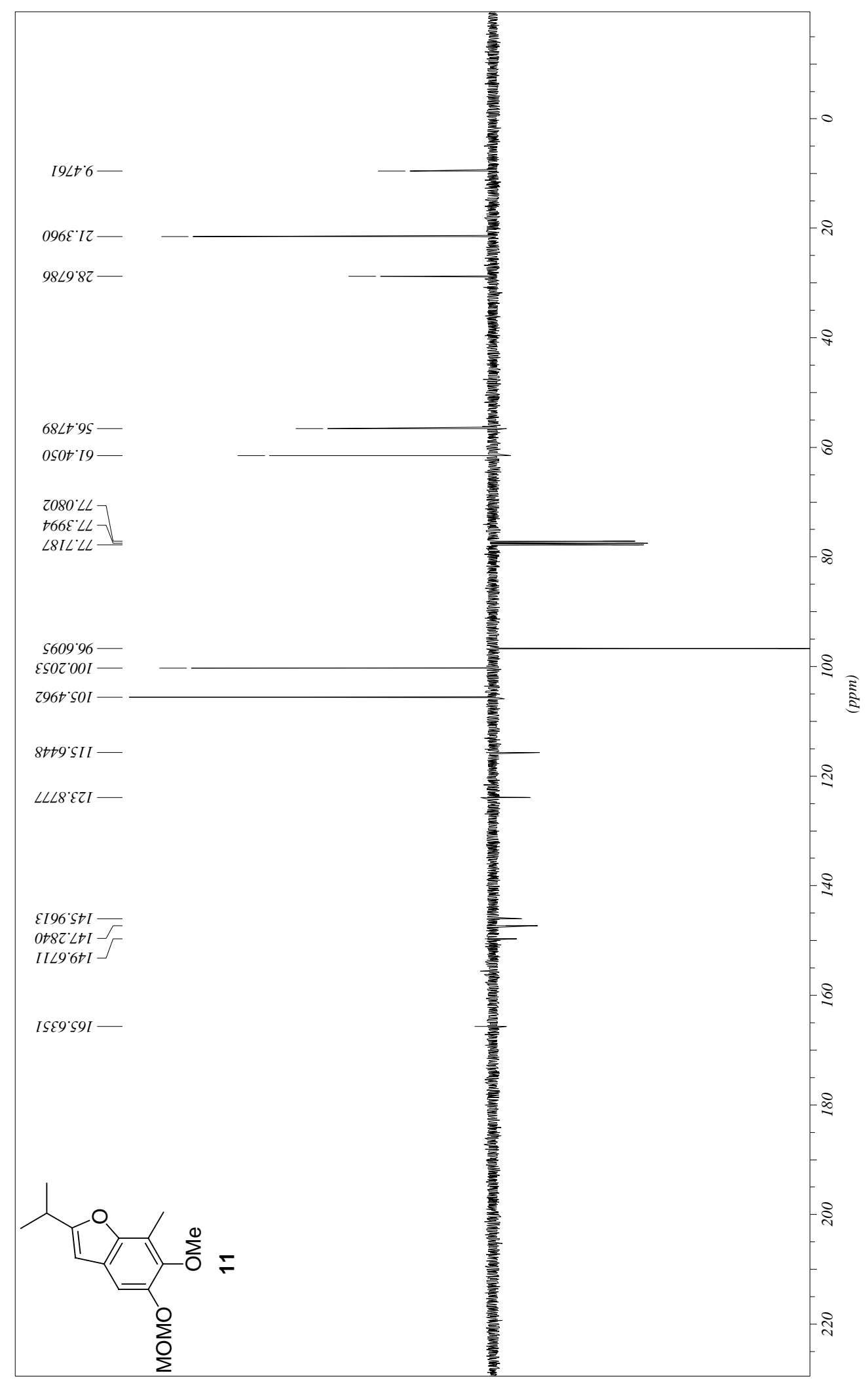




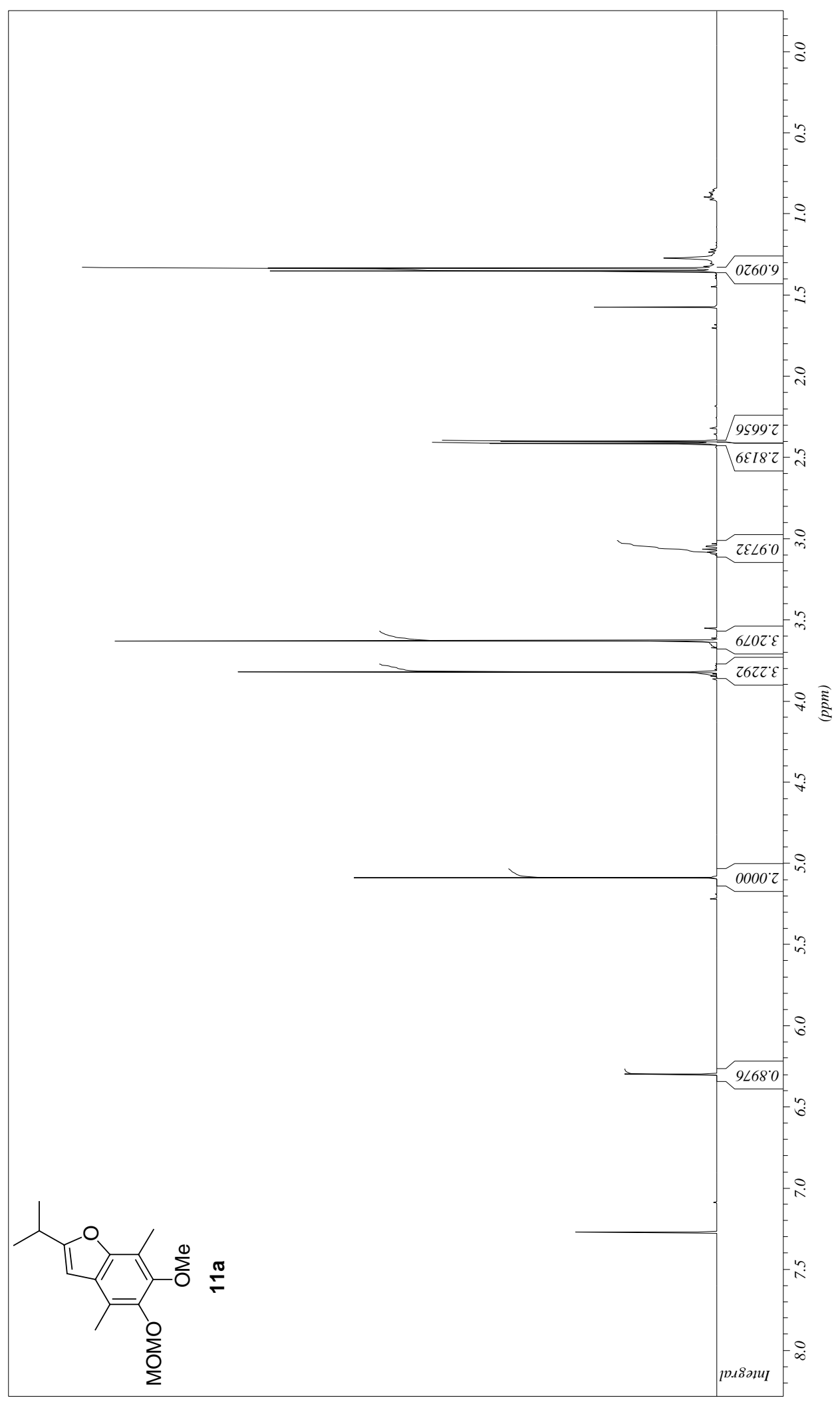




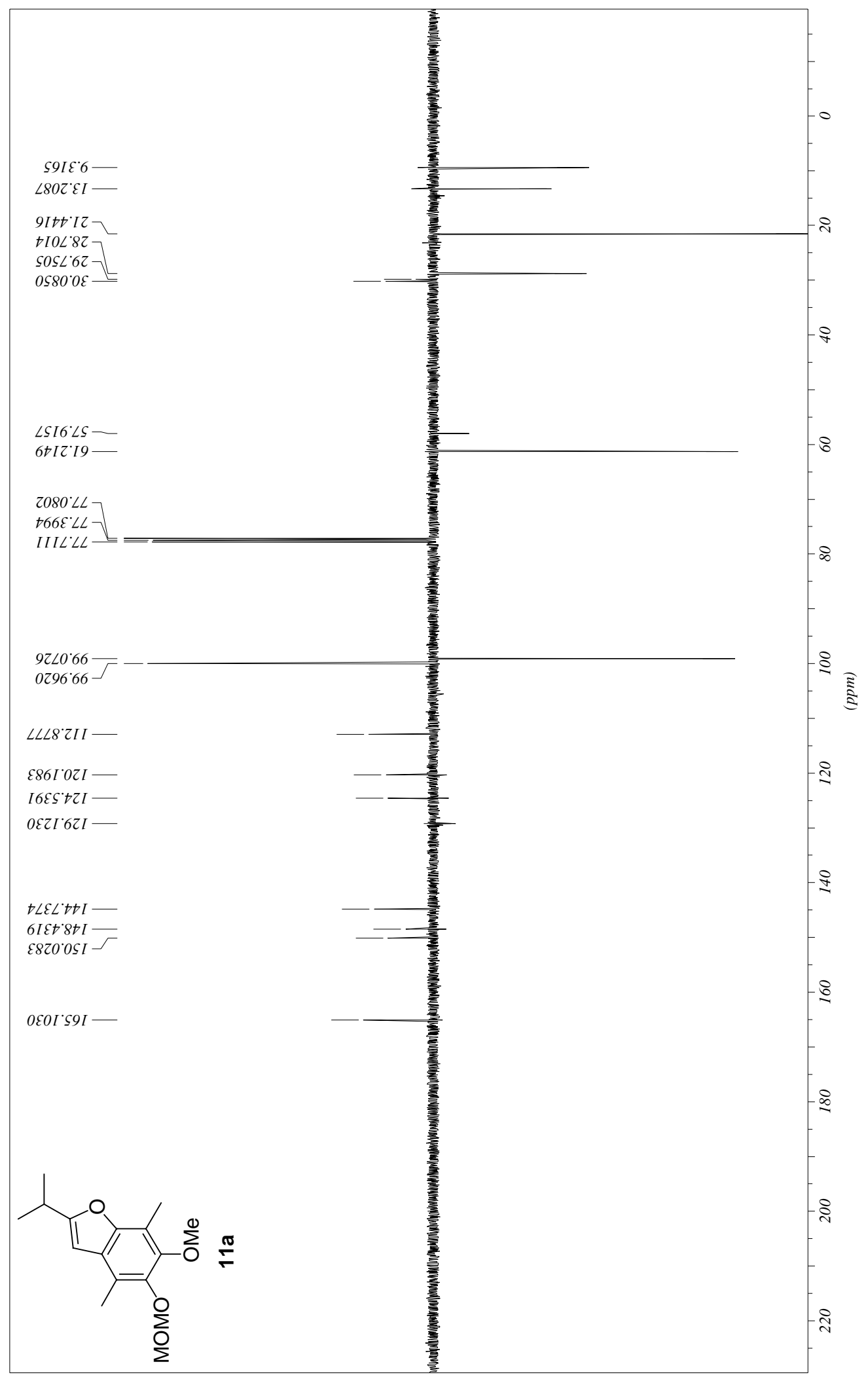




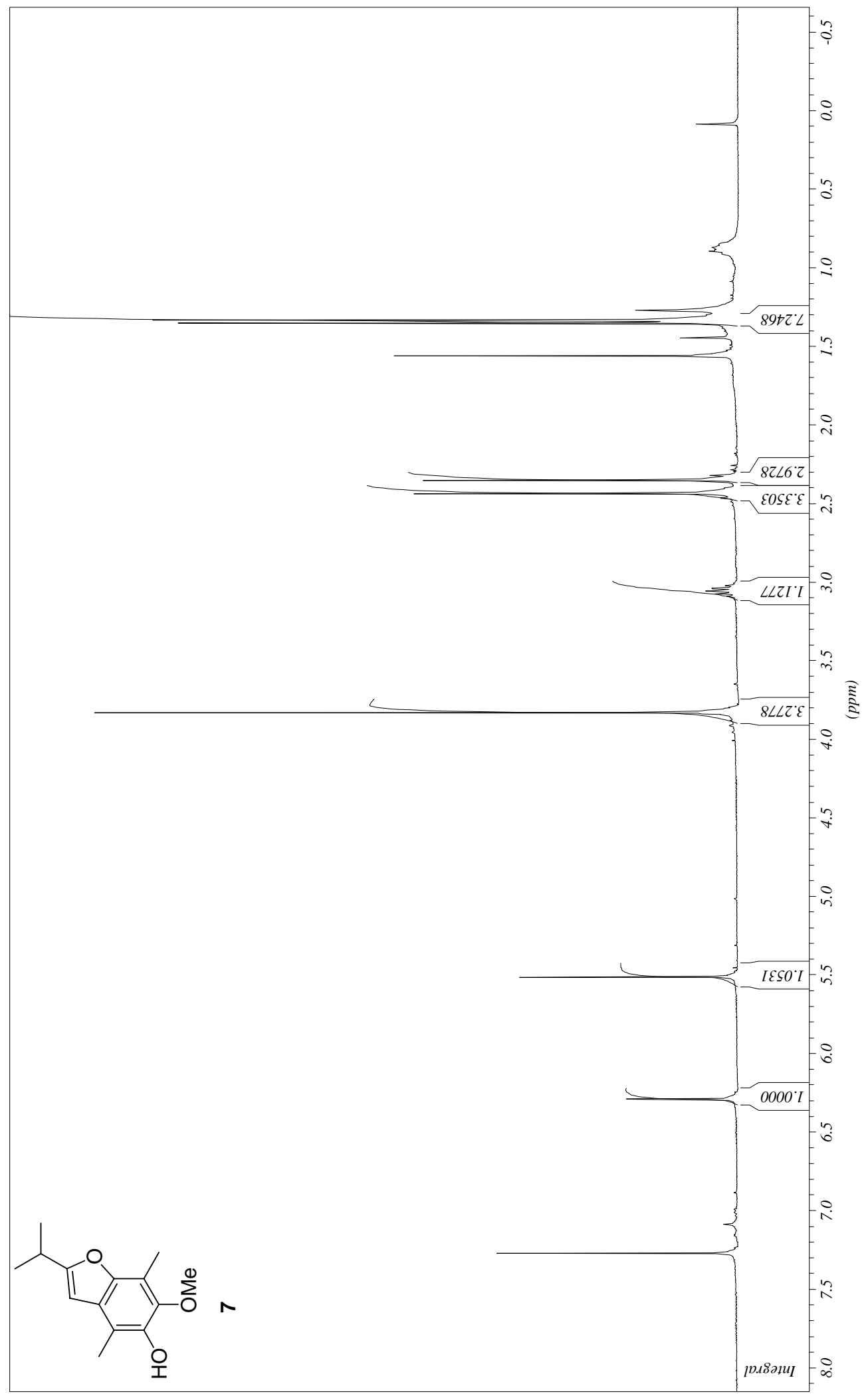

ิㅗำ 


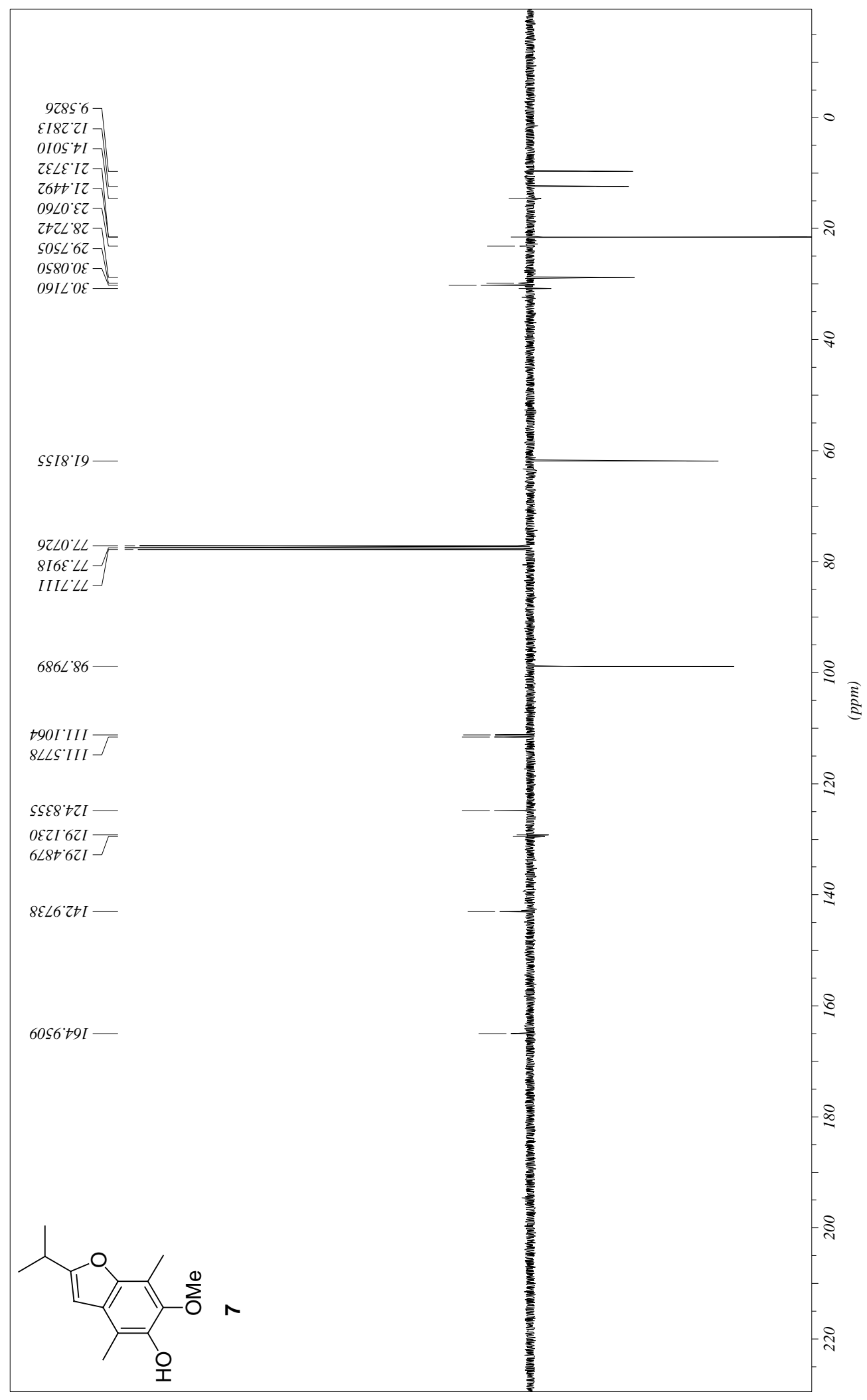




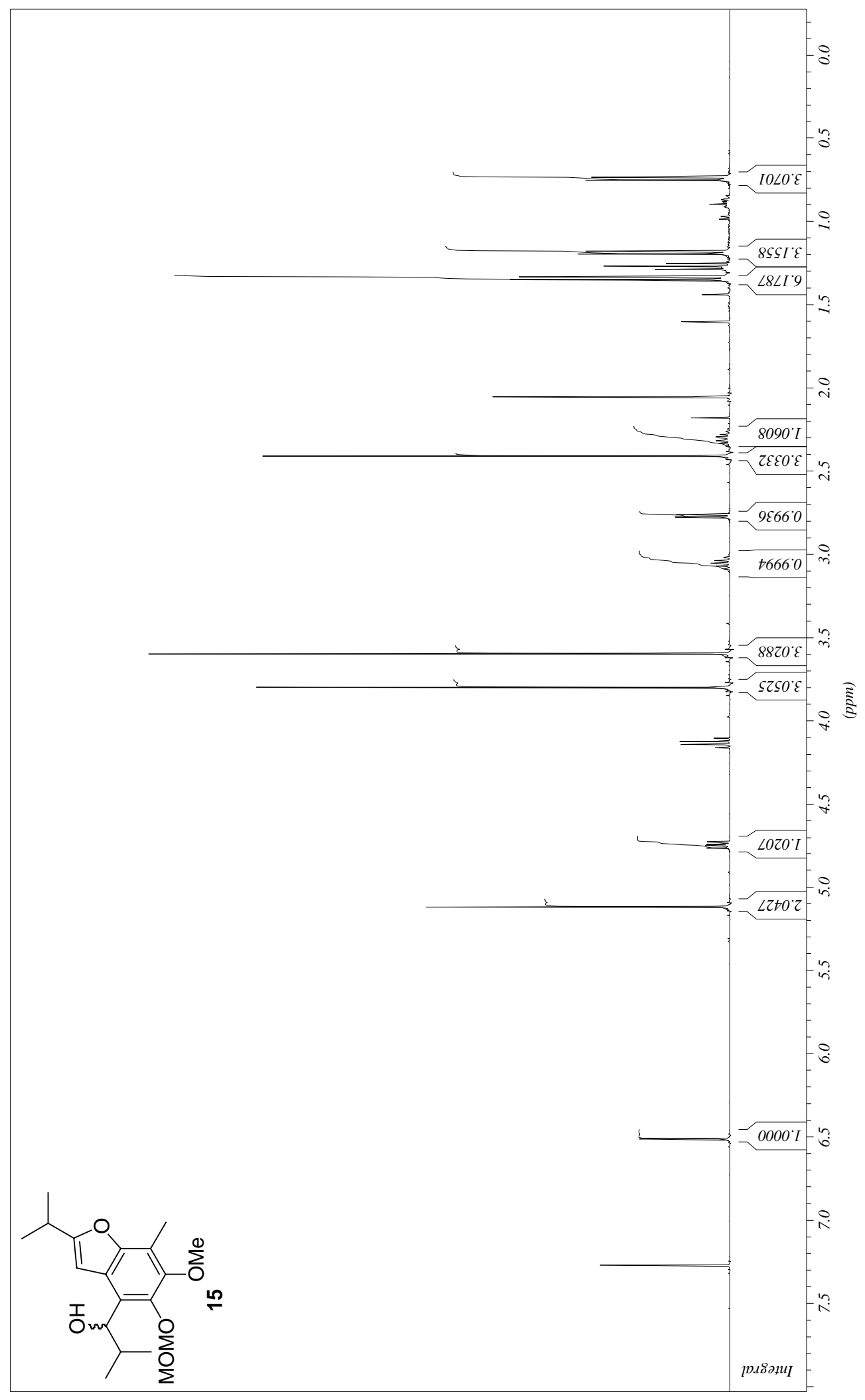




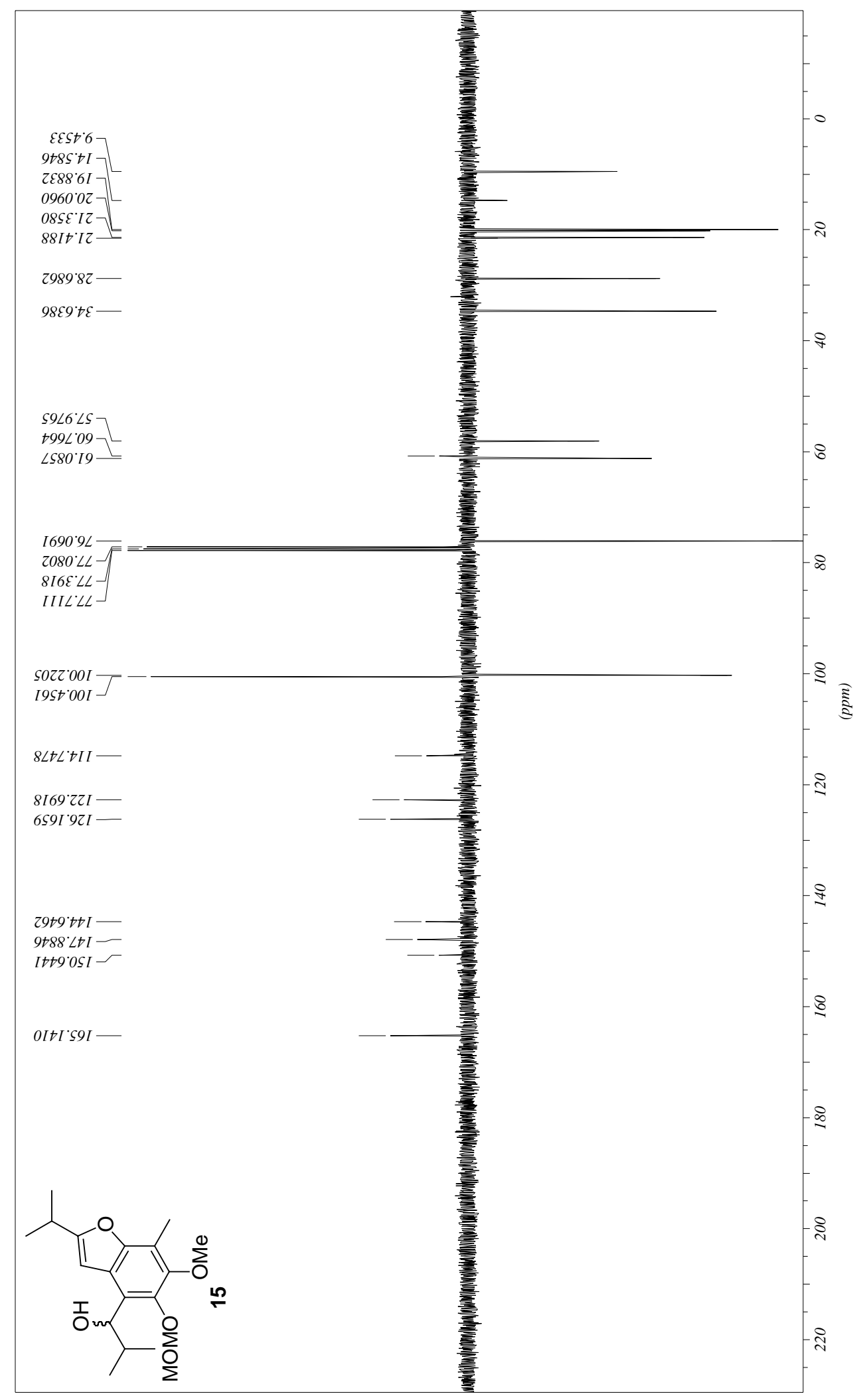




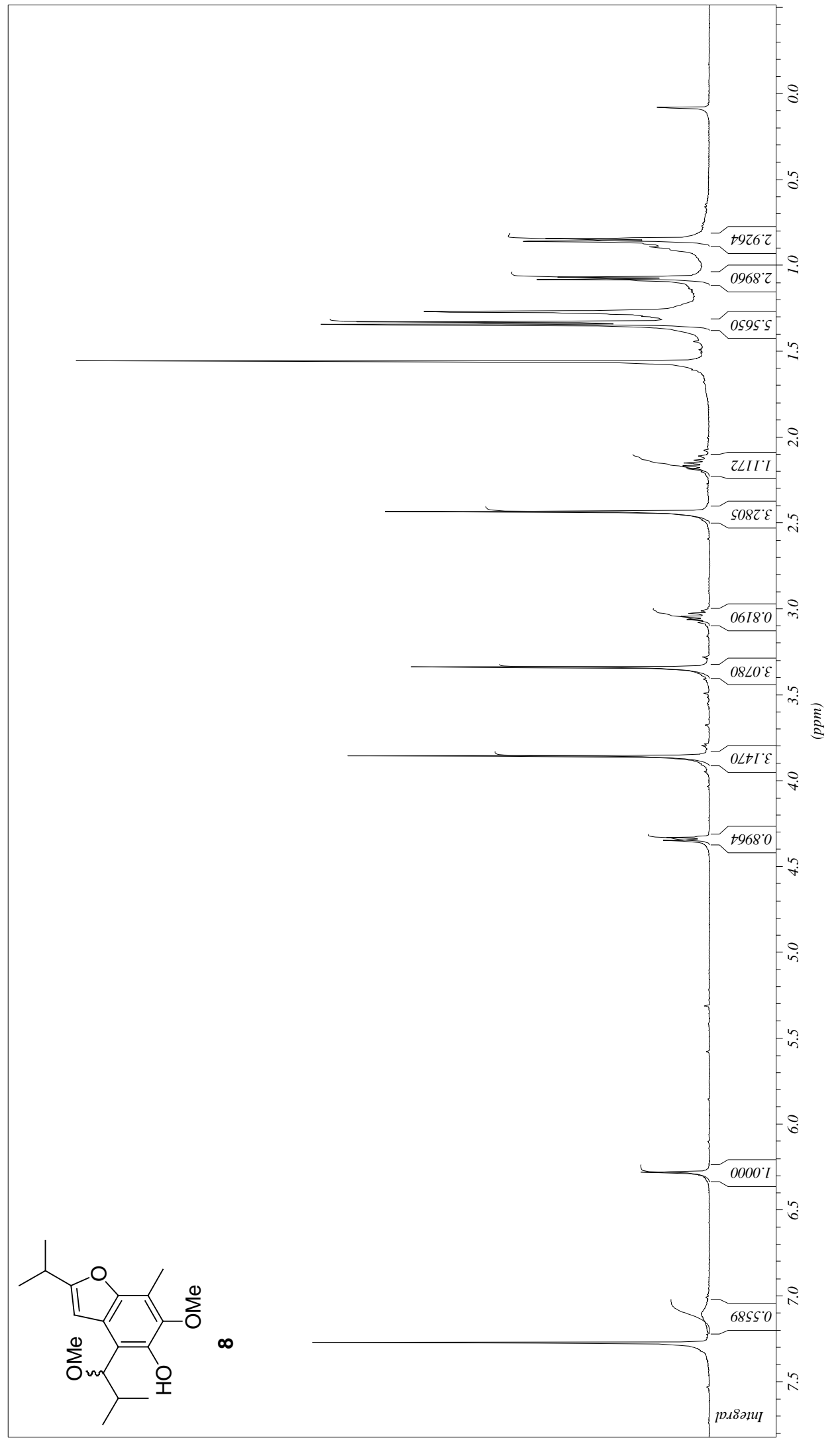




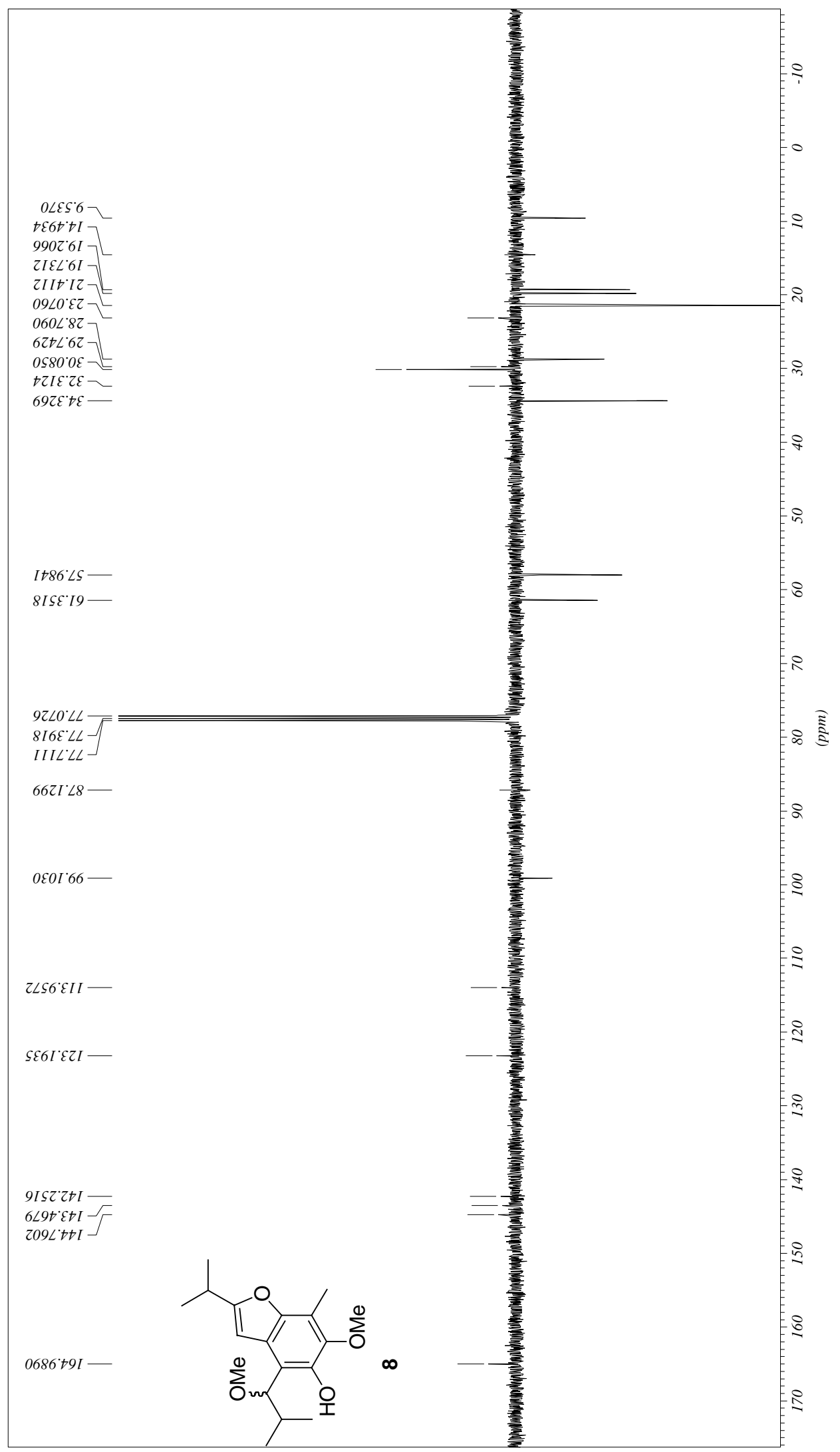




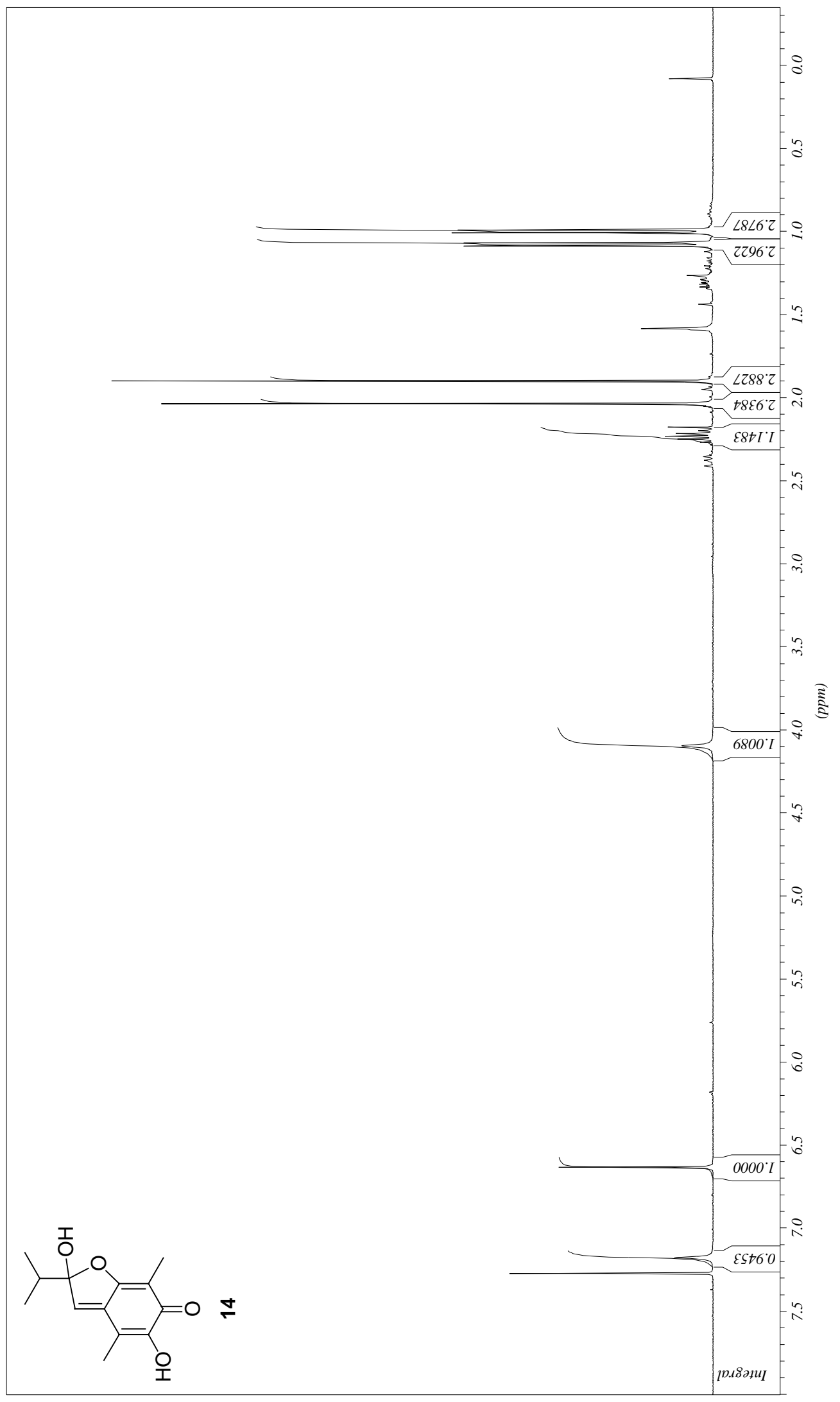




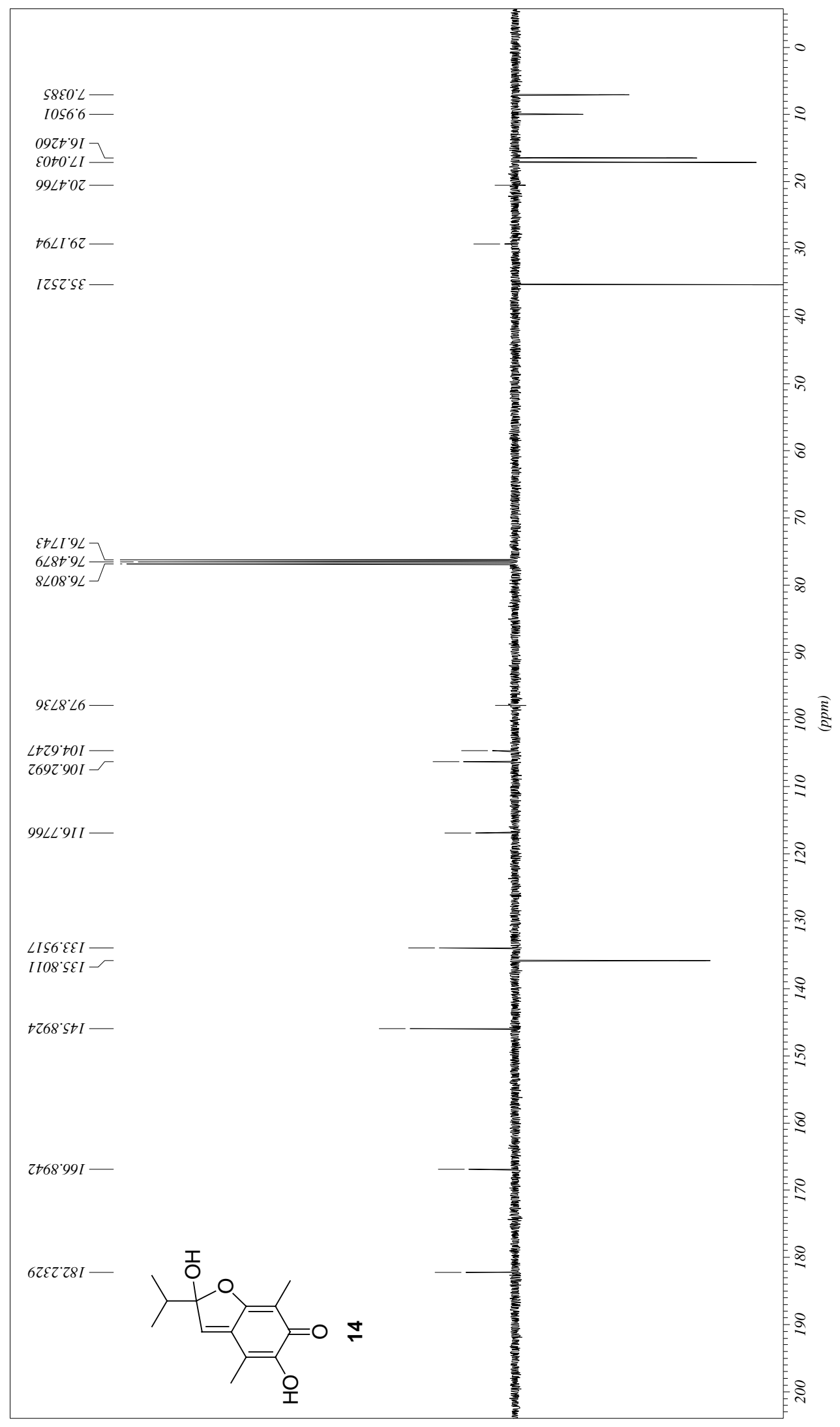




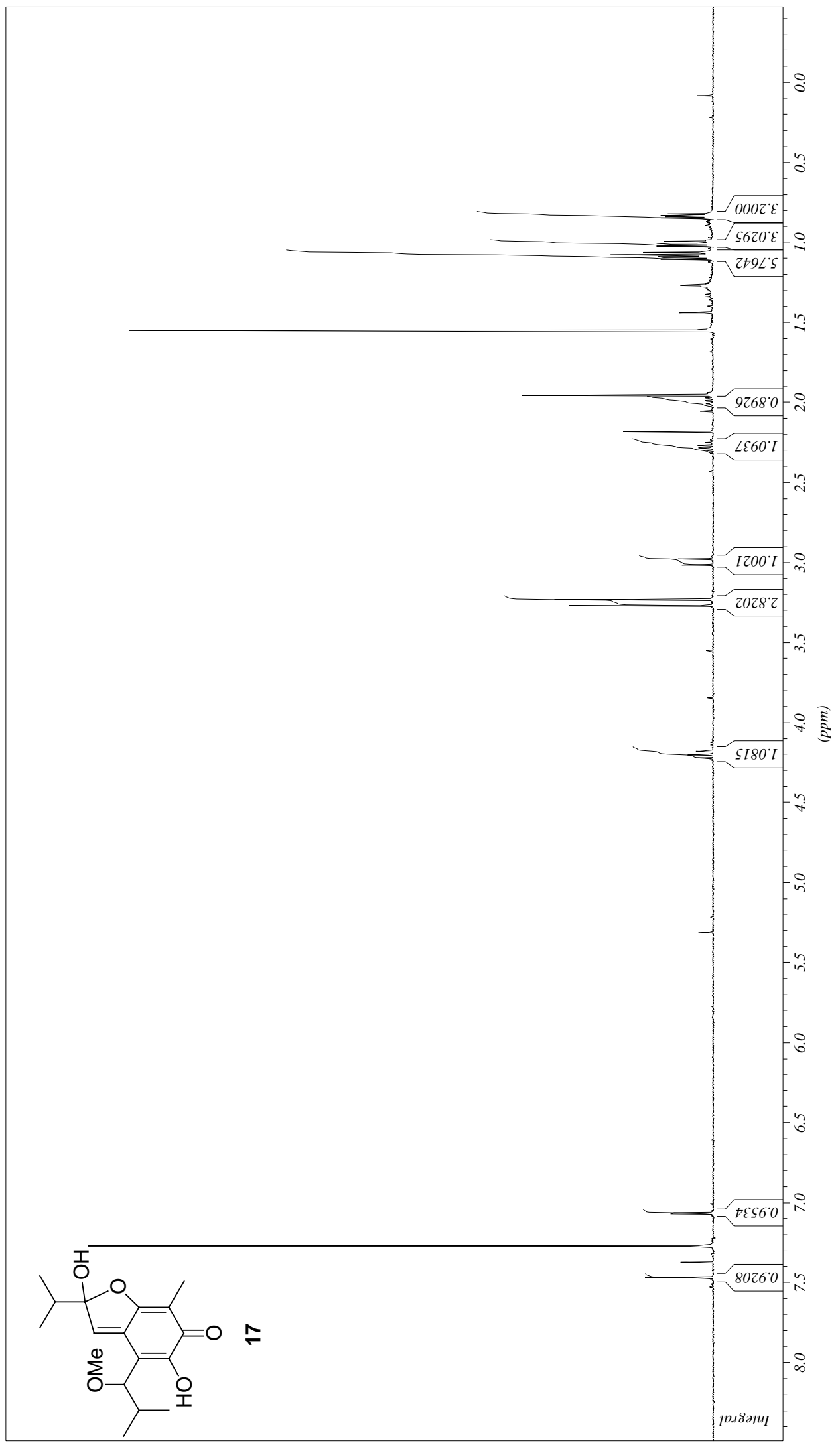




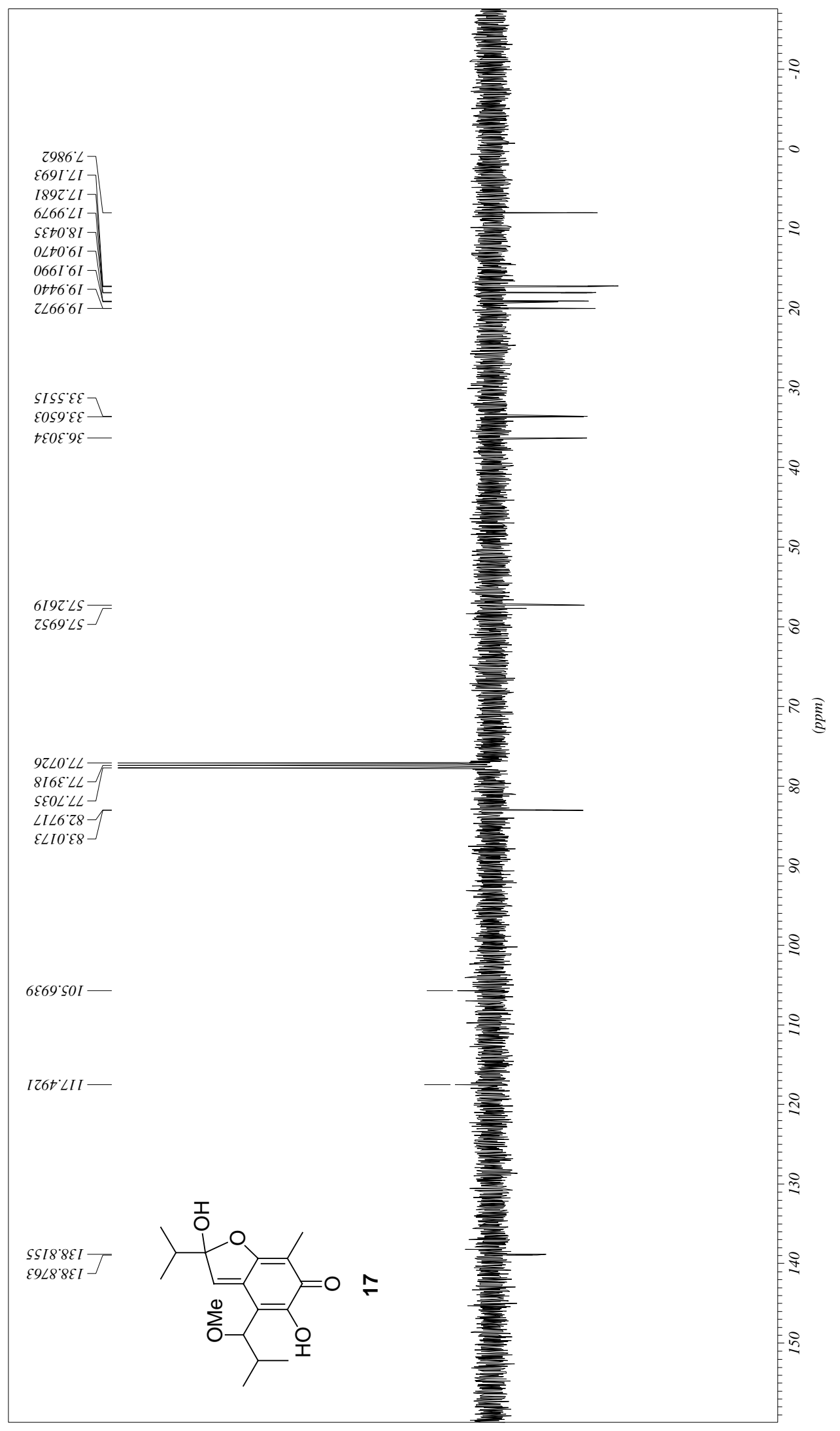

